TITLE:

\title{
Modeling shared autonomous electric vehicles: Potential for transport and power grid integration
}

\section{$\operatorname{AUTHOR}(S)$ :}

lacobucci, Riccardo; McLellan, Benjamin; Tezuka, Tetsuo

\section{CITATION:}

Iacobucci, Riccardo ... [et al]. Modeling shared autonomous electric vehicles: Potential for transport and power grid integration. Energy 2018, 158: 148-163

\section{ISSUE DATE:}

2018-09-01

URL:

http://hdl.handle.net/2433/241777

\section{RIGHT:}

(C) 2018 This manuscript version is made available under the CC-BY-NC-ND 4.0 license

http://creativecommons.org/licenses/by-nc-nd/4.0/; The full-text file will be made open to the public on 1 September 2020 in accordance with publisher's 'Terms and Conditions for Self-Archiving'; この論文は出版社版でありません。引用 の際には出版社版をご確認ご利用ください。; This is not the published version. Please cite only the published version. 


\title{
Modeling Shared Autonomous Electric Vehicles: potential for transport and power grid integration
}

\author{
Riccardo Iacobucci*, Benjamin McLellan, Tetsuo Tezuka \\ Graduate School of Energy Science, Kyoto University, Yoshida Honmachi, Sakyo-ku, \\ Kyoto 606-8501, Japan
}

\begin{abstract}
One-way car-sharing systems are becoming increasingly popular, and the introduction of autonomous vehicles could make these systems even more widespread. Shared Autonomous Electric Vehicles (SAEVs) could also allow for more controllable charging compared to private electric vehicles, allowing large scale demand response and providing essential ancillary services to the electric grid. In this work, we develop a simulation methodology for evaluating a SAEV system interacting with passengers and charging at designated charging stations using a heuristic-based charging strategy. The influence of fleet size is studied in terms of transport service quality and break-even prices for the system. We test the potential of the system to supply operating reserve by formulating an optimization problem for the optimal deployment of vehicles during a grid operator request. The results of the simulations for the case study of Tokyo show that a fleet of SAEVs would only need to be about 10\%-14\% of a fleet of private cars providing a comparable level of transport service, with low break-even prices. Moreover, we show that the
\end{abstract}

\footnotetext{
${ }^{*}$ Corresponding author. E-mail: iacobucci.riccardo.38e@kyoto-u.jp
} 
system can provide operating reserve under several operational conditions even at peak transport demand without significant disruption to transport service.

Keywords: autonomous vehicles, electric vehicles, mobility-on-demand, operating reserve, vehicle-to-grid, demand response

\section{Introduction}

The ubiquitous presence of the internet and smartphones is allowing a shift from car ownership to intelligent car sharing models of transportation. One-way car sharing services (in which cars can be taken wherever they are currently parked and left at any other place within a specified area) are already commonplace in large cities in Europe [1]. The diffusion of shared transportation can significantly change the vehicle ownership rateand improve the efficiency of the transport sector: each car sharing vehicle is estimated to remove 9 to 13 vehicles from the roads [2], as most private cars are used less than $10 \%$ of the time [3]. It can also improve the efficiency of the transport sector, as high annual vehicle-km traveled per vehicle create a strong economic incentive towards highly efficient vehicles [2]. Shared transport is also expected to be cheaper than private transportation. Savings for the average American household are estimated to be about $\$ 6,000$ a year by joining a shared transport program instead of owning a private vehicle [4].

The advent of autonomous driving technology will further speed up the adoption of this transport mode, making it cheaper, more convenient, and more suitable to be used efficiently in less densely populated areas [3]. more 
convenient: vehicles can move to pick up customers autonomously without the need to move to a parked vehicle, a service comparable to that of a taxi without the cost of the driver. The popularity of similarly convenient but relatively expensive services such as Uber show the potential for this kind of transport mode. Autonomous vehicles have been extensively tested and are planned to be commercially available by the next decade [5]. Advantages of a car sharing system using autonomous vehicles include the efficiency gains from automated driving(more precise and less wasteful driving, less incidents), with fuel economy improvements of 4 to $10 \%$, elimination of the time spent for parking, and decreased need for parking spaces in cities [4]. Autonomous vehicles also have the potential to significantly decrease greenhouse-gas emissions in the transport sector, with estimated 87\%-94\% decreases in per-mile GHG emissions compared to current private vehicles in the United States by 2030 mainly due to the possibility of using vehicles sized to the specific trip [6].

Shared autonomous vehicles can also facilitate the electrification of the transport sector, as the cars involved can optimize their state of charge (SOC) and their charging schedule while reliably ensuring service to the user [7]. This can overcome several problems currently hindering the wider adoption of electric vehicles, such as the scarcity of charging stations [8], high cost, and range limitations [9]. It is therefore important to study the impact of this system on the electricity grid [10]. This type of car sharing-using autonomous driving technology and battery electric vehicles - will be referred to as Shared Autonomous Electric Vehicles (SAEV) in this work.

It is predicted that the widespread adoption of electric vehicles could 
significantly change the management and balancing of the electricity system and facilitate the integration of intermittent renewable energy in the grid [11]. For example Dallinger and Wietschel [12] found that EVs can absorb over $50 \%$ of the yearly excess renewable generation in the high renewable scenario of Germany in 2030 that would have been curtailed otherwise. Electric vehicles with vehicle-to-grid (V2G) power capability can also offer several additional services to the electric grid, such as peak power generation, operating reserve, and regulation [13]. Implementation of V2G can also allow a higher renewable energy penetration by further increasing grid flexibility [14]. However, private electric vehicles are expected to put a large burden on distribution systems, especially when considering large scale V2G implementation [15]. By contrast, SAEVs can be more easily controlled and optimized to implement fast, large-scale demand response [16]. This would allow a deeper grid integration, which is fundamental to achieving the potential environmental benefits of vehicle electrification [17]. SAEVs would also allow easier utilization of electric vehicles for providing ancillary services, which may be uneconomical for private vehicles [18]. Another potential advantage of SAEVs is their ability to move autonomously to specific charging stations. This allows for a direct connection to the high voltage electricity transmission system in designated points without overloading the low-voltage distribution network. The centralization of grid connection may also help to implement efficient $\mathrm{V} 2 \mathrm{G}$ connections by providing more balanced and controlled electricity flow and improved safety equipment.

Previous work on the impact of car sharing systems and SAEVs has mostly focused on the transport implications, without considering the impact 
on the electric grid. In this work, a system of autonomous electric vehicles is considered and its interaction with the power grid is investigated in terms of charge scheduling and ability to supply operating reserve. It is assumed that vehicles are managed by a company or entity that oversees the shared transport service. Control is of two types: the vehicle routing control is centralized, with a central authority (control center) assigning available vehicles to new passenger trips: the decision to move to charging station, and how much to charge, is decentralized, and depends on each vehicle's SOC, waiting times, and the current and expected price of electricity and aggregated transport demand. The vehicles are assumed to communicate with the control center reporting their status and SOC in order for the control center to optimize the assignment of trips. In case of grid requests, the charging decisions are centralized and controlled by the control center to reach the aggregated power generation needed.

The work includes a static transport model based on transport survey data to simulate the transport patterns. Data from a transport survey for the city of Tokyo was used as a case study. The vehicles satisfy trip requests while charging their batteries according to a heuristics-based demand-response strategy based on electricity price signals from the grid. The potential of the system to participate in the operating reserve market and the influence of several parameters on the results were tested.

The aim of this work is first to evaluate the feasibility of the autonomous car sharing system in terms of transportation service quality and economic performance. Subsequently, a new system for responding to grid capacity requests is proposed and tested to evaluate the SAEV system performance 
as an operating reserve provider. In the system, the fleet of SAEVs provide spinning and non-spinning operating reserve in response to grid operator requests (both to generate energy and to absorb surplus generation). The results show that a $\mathrm{SAEV}$ system is economically viable in terms of transport service even at low request densities, and can be used reliably as an emergency supply of energy when coordinated with the electric grid.

The work is organized as follows. In section 2 the existing literature on the topic is reviewed. In section 3 the model proposed is presented. This includes the transport model, the charge scheduling algorithm, and the operating reserve model. The model evaluation criteria and the assumptions and limitations of the study are discussed. The methodology related to the case study is also presented in this section. In section 4 the results of the simulations for the case study are discussed. This section is divided into two parts: in the first part the optimal fleet size and the system's break-even prices are determined; in the second part the ability of the system to provide operating reserve under a variety of scenarios using the findings of the first part is investigated. In section 5 the conclusions are reported.

\section{Related work}

Previous work on the impact of car sharing systems and SAEVs has mostly focused on the transport implications, without considering the impact en the electric gridThis section provides a summary of previous work on shared autonomous electric vehicles, which has mainly focused on transport aspects. Burns et al. [19] simulate a fleet of shared autonomous vehicles (SAV) fulfilling the transport demand of a city in which numerical and 
analytical models were developed with simplified assumptions, such as homogeneous trip rates and simplified distance calculations without a road network. The model was applied to several case studies in different contexts, and the authors concluded that in all cases SAVs offered higher efficiencies, lower costs and higher convenience to users, when compared to other public and private transport modes. In the specific case study of Manhattan, it was found that 9,000 SAV could replace over 13,000 taxicabs by satisfying the same transport demand with a total cost of $0.31 \$ / \mathrm{km}$, compared to $2.5 \$ / \mathrm{km}$ for current taxis, while decreasing waiting times from an average of 5 minutes to 1 minute. Fagnant and Kockelman [3] also developed an agent-based model of SAV using simplified transport assumptions. Macro areas with homogeneous trip generation rates and gridded road network were used. They concluded that SAV could provide adequate service with a fleet size of about a tenth of the equivalent fleet of private vehicles, and that the quality of service was dependent on the density of users. In [20], the transport modeling framework MATSim was employed to predict the impact of SAV on the modal share of the transport sector. On a simplified road network, the results showed that SAV could be the dominant transport mode, potentially also disrupting public transport. Liu et al. [21] used MATSim to simulate a fleet of SAV in Austin, Texas, to investigate the rate of penetration of SAV at different price levels. They found that mode split reaches over $50 \%$ with a $0.31 \$ / \mathrm{km}$ fare. The authors did not consider changes in transport patterns due to SAVs, but only change in mode split for the same trips. Other authors have explored the impact of SAV on urban form, including urban parking demand, suggesting that SAV could eliminate the need for 
$90 \%$ of current parking space for users of the system [22].

Spieser et al. [23] used a more detailed and realistic transport model to study the fleet sizing problem and estimate the economic benefits of a fleet of SAV replacing all other private transport modes in Singapore, based on actual transport data. The authors determined both the minimum fleet size to meet the transport demand of the city and the fleet size necessary to obtain a certain peak waiting time. The results show that, for the specific case of Singapore, the personal mobility needs of the entire population can be met with a fleet size of a third of the total number of passenger vehicles currently in operation. This work also assumes unchanged transport patterns.

Levin et al. [24] focused on studying the effect of SAVs on traffic congestion by introducing SAVs in existing traffic simulation models. The results show that the level of service of SAV may be lower than predicted by previous studies when accounting for traffic congestion, since SAV may shift demand from other modes and increase the number of passenger-km traveled by car. However, they found that ride-sharing (more passengers sharing the same vehicle) was effective at solving this problem. Moreover, differential pricing (peak price) may also be beneficial in limiting peak congestion.

Several other studies have dealt with the problem of shared autonomous vehicle rebalancing strategies [25, 26, 27], which is however not considered in this work. In all these studies the energy aspects were not considered, and the vehicle energy source was generally not specified.

Some studies also considered the charging constraints of electric vehicles, however they assume uncoordinated charging. Zhang et al. [28] developed a model predictive control approach for the optimization of an autonomous 
car-sharing system with rebalancing which considers electric charging constraints. The problem is solved as a mixed integer linear program (MILP). Although The model does not consider the position of charging stations and assumes uncontrolled charging of vehicles. Furthermore, although their approach is optimal within the model assumptions, the MILP approach to the problem make the model not scalable to systems with large number of nodes.

Rigas et al. developed a mixed integer programming optimization for shared electric vehicles with battery swapping [29]. Biondi et al. [30] propose an optimization formulation for the positioning of charging station for electric car sharing systems and analyze the impact of these fleets on the electricity grid.

Chen et al. [7] studied the operation of a SAEV system with a model based on [3]. The agent-based transport model methodology is similar, but the investigation is expanded by including charging of the electric vehicles serving $10 \%$ of trip demand in a medium-sized metropolitan area. The analysis includes a charging station generation phase to find the number and position of charging stations needed to serve passengers within a certain waiting time. The model was run in different scenarios to investigate the sensitivity to several parameters. The study considers short- and long-range type of vehicles, with slow and fast charging. It was found that although double the number of vehicles are needed for the case with short-range and slow charging vehicles, this is the most profitable scenario. For the case study in Austin, Texas, the results indicate that each SAEV can replace between 5 and 9 private vehicles, depending on range and speed of charge. The model 
does not consider 'smart' charging and found that simultaneous charging of the fleet at peak times may be problematic for the electric grid.

Shared non-autonomous electric vehicles (car sharing systems) have also received much interest in recent years, and there are a number of studies focused on the integration of these vehicles with the electricity system. Freund et al. [31] developed a control and optimization system to manage the charging of shared electric vehicles in a smart microgrid in order to maximize the use of renewable energy sources. In another study [1], a model of an electric vehicle car sharing system with reservation was developed. The model was based on charging stations serving requests in the vicinity. The fact that demand is known in advance through reservation allows for the use of an optimization algorithm, which is also used to determine the optimal fleet size by maximizing the car sharing operator's net revenues and the user's benefit, also taking into account the necessary car relocations among charging stations. Several authors explore the feasibility of taxi services using electric vehicles. Bischoff and Maciejewski [32] studied a fleet of electric (nonautonomous) taxis through MATSim. The authors conclude that electric vehicles can be used as taxis and only a limited number of charging pods is sufficient. However, the work does not focus specifically on the grid-side aspects. In another study [33], the operation of a electric taxi fleet with trip reservation in Singapore was investigated. An interesting aspect of the results is that changing the number of charging stations had limited effect on the performance of the system.

The use of electric vehicles as grid service providers has been studied extensively [34], demonstrating the feasibility and effectiveness of using these 
vehicles for grid stabilization and to replace fossil fuel power plants in grids with high penetration of renewable energy [35]. However, these studies all focus on private vehicles.

In summary, while there have been a number of studies considering aspects of electric vehicle systems - some as shared taxis, some looking at charging-system balancing - it is apparent that these have not covered the situation of shared autonomous electric vehicles operating as both transport and grid storage / ancillary services provider under situations with minimal advance knowledge of the transportation required (i.e. no reservation). The present study therefore seeks to address this gap in knowledge.

\section{Nomenclature}

$\begin{array}{ll}\text { A. Indices } & \\ h & \text { Hour } \\ k & \text { Trip } \\ t & \text { Time step } \\ v & \text { Vehicle } \\ B . \text { Simulation variables } \\ A_{i} & \text { Area of node } i \\ a_{v}(t) & \text { Waiting time variable } \\ B E P & \text { Break-even price } \\ d_{i, j} & \text { Distance between node } i \text { and } j \\ e_{v}(t) & \text { Energy exchanged } \\ f(h) & \text { Frequency of trips at hour } h \\ \ell & \text { Length of time step (minutes) } \\ m(t) & \text { Price of electricity } \\ p_{v}(t) & \text { Charging power } \\ q_{v}(t) & \text { State of charge (SOC) of battery }\end{array}$




\begin{tabular}{|c|c|}
\hline$r d_{k}$ & Destination node of trip $k$ \\
\hline$r o_{k}$ & Origin node of trip $k$ \\
\hline$r t_{k}$ & Time (hour) of trip $k$ \\
\hline$r w_{k}$ & Weight of trip $k$ \\
\hline${ }^{i} s_{v}$ & Binary state variable $i$ \\
\hline$T$ & Total number of time steps \\
\hline$T P H$ & Average trips per hour \\
\hline$V$ & Total number of vehicles \\
\hline$w_{v}(t)$ & Distance to current destination \\
\hline$W_{\text {pass }, v}$ & Total distance traveled with passengers \\
\hline$W_{t o t, v}$ & Total distance traveled \\
\hline$\lambda(t)$ & Expected number of requests at time step $t$ \\
\hline$\Delta_{\max }$ & Price of electricity at which car charge at maximum rate \\
\hline$\pi_{v}(t)$ & Agent price \\
\hline \multicolumn{2}{|c|}{ C. Operating reserve simulation variables } \\
\hline$r_{\max }(t)$ & Total power available for request at time $t$ \\
\hline$\alpha_{v}$ & Initial delay of $v$ before connection \\
\hline$\Gamma$ & Duration of the request \\
\hline$\delta$ & Allowed delay of request \\
\hline$\epsilon_{v}(\tau)$ & Energy delivered by $v$ at minute $\tau$ \\
\hline$\tau$ & Time step \\
\hline \multicolumn{2}{|c|}{ D. Parameters and constants } \\
\hline$a_{\text {con }}$ & Time needed to connect to charging station \\
\hline$a_{\text {idle }}$ & Maximum idle time \\
\hline$a_{\text {charge }}$ & Minimum charging time \\
\hline$C_{\text {battery }}$ & Cost of battery \\
\hline$C_{c a r}$ & Cost of car (with no battery) \\
\hline$C A P$ & Battery capacity (kWh) \\
\hline$E C$ & Electricity consumption of cars $(\mathrm{kWh} / \mathrm{km})$ \\
\hline$h z$ & Prediction horizon \\
\hline
\end{tabular}




\begin{tabular}{|c|c|}
\hline$L_{\text {battery }}$ & Life of battery in equivalent full cycles \\
\hline$L_{\text {car }}$ & Life of car not including battery (years) \\
\hline$p_{i, j}(t)$ & Probability of a trip starting in $i$ with destination $j$ \\
\hline$p_{\max }$ & Maximum charge rate $(\mathrm{kW})$ \\
\hline$p_{\text {peak }}$ & Peak charge rate for short periods $(\mathrm{kW})$ \\
\hline$q_{\text {charge }}$ & SOC at which car move to charging stations \\
\hline$q_{\max }$ & Maximum SOC in normal operation \\
\hline$q_{\min }$ & Minimum SOC in normal operation \\
\hline$u(t)$ & Average speed of vehicles (km/time step) \\
\hline$P S P$ & Passenger service priority factor (JPY $/ \mathrm{km}$ or USD $/ \mathrm{km}$ ) \\
\hline$\beta$ & Ratio of trip distance to Euclidean distance \\
\hline$\eta$ & Battery round-trip efficiency \\
\hline \multicolumn{2}{|c|}{ E. Acronyms } \\
\hline JPY & Japanese Yen \\
\hline $\mathrm{SAV}$ & Shared autonomous vehicle \\
\hline SAEV & Shared autonomous electric vehicle \\
\hline $\mathrm{SOC}$ & State of charge \\
\hline TOD & Time of day pricing \\
\hline USD & U.S. Dollars \\
\hline $\mathrm{V} 2 \mathrm{G}$ & Vehicle to grid \\
\hline
\end{tabular}

\section{Methods}

This section describes the methods used in the work. The first part of the section deals with the general methodology for the transport model and the operating reserve request model. In the second part, the specific case study methods are reported. These include the calculation of the parameters used. 


\subsection{Transport model}

The first, and fundamental component of this study, was the transport route and trip selection model. This is used to determine where SAEVs travel, which is primarily in order to satisfy customer requests. The model applied in this study was developed in MATLAB ${ }^{\mathrm{TM}}$ and is based on a simplified road network, represented by nodes at specific coordinates and their associated areas.

The simulation evolves through $T$ time steps. At each time step, trip requests can arrive at each node of the model, with an associated destination node. A fleet of $V$ autonomous electric vehicles move from one node to another satisfying trip requests.

In order to have an acceptable computational time for the simulation, the actual street layout is not considered. The distance is calculated as the Euclidean distance times a tortuosity factor $\beta$ that represents the lengthening due to the city's street layout. The distance between nodes is stored in a distance matrix where each element $d_{i, j}$ represents the distance between node $\mathrm{i}$ and node $\mathrm{j}$.

Distances inside the same node (for trips starting and ending in the same node) are calculated using the approximation of the average distance between two uniformly distributed random points in a square:

$$
d_{i, i}=0.52 \cdot \sqrt{A_{i}} \cdot \beta
$$

Where $A_{i}$ is the area associated with node $i$.

These assumptions do not alter the probabilistic location of requests' origins or destinations, as these are the initial given conditions of the model. 
They do, however, fail to consider the congestion effect and the fact that a real road network is not homogeneous. This is considered acceptable for this work, as the aim is not to simulate the change in the city traffic patterns, but to understand the energy aspects of SAEV as a 'marginal' player (that does not significantly alter the transport patterns) in the transport system. This also makes the model readily adjustable to alternative cities if the other required data is available. It is, however, important to consider the average effect of traffic congestion, because it can significantly change the pattern of availability of vehicles during peak times. Traffic congestion is therefore introduced in the model as a variable average speed of vehicles. This is represented with a periodic time-varying vector $u(t)$ which represents the distance traveled by each vehicle in a time step of the simulation. This is related to the average speed of vehicles in $\mathrm{km} / \mathrm{h}$ by a factor $\ell / 60$.

Table 2: States of vehicles

\begin{tabular}{ll} 
state & eondition-description \\
\hline 0 & charging, not available \\
1 & charging, available \\
2 & idle, available \\
3 & moving, available after drop-off \\
4 & moving to charging station, not available \\
5 & connecting to charging station, not available
\end{tabular}

The current state of each vehicle $v$ is registered as a binary variable ${ }^{i} s_{v}(t)^{i} s_{v}(t) \in\{0,1\}$, representing different situations. If the vehicle is currently in state $i$, the corresponding state variable ${ }^{i} s_{v}(t)$ is set to 1 , otherwise 
it is set to 0. A summary of the different states is presented in Table 2 and Fig. 1. Vehicles can be in only one state at any given time:-

$$
\sum_{i=0}^{5}{ }^{i} s_{v}(t)=1
$$

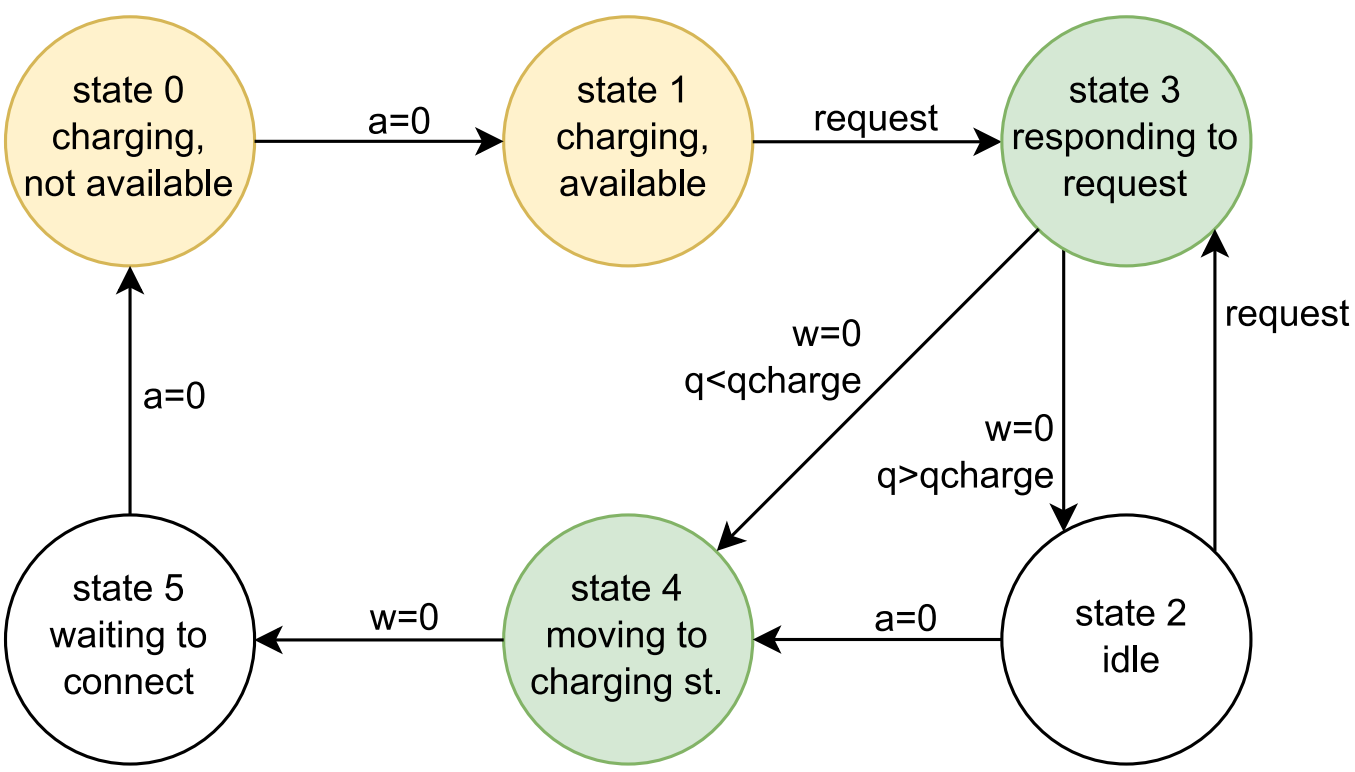

Figure 1: Simplified outline of the model. Yellow backgrounds indicate connection to the grid, green backgrounds indicate movement.

\subsection{Trip requests}

Trip requests are generated stochastically during the simulation. The number of requests at each time step $t$ is decided through a Poisson process with a periodically time-varying rate $\lambda(t)$. The number of requests in each time step $t$ is therefore sampled from the Poisson distribution with a cumulative mass function $(\mathrm{CMF})$ defined as: 


$$
C M F(x)=\sum_{i=1}^{x} \frac{\lambda(t)^{i} \cdot e^{-\lambda(t)}}{i !}
$$

Each request is then associated with a starting node and a destination node $[i, j]$. The origin/destination pair is extracted from a periodically timevarying distribution where $p_{i, j}(t)$ is the probability associated with the pair $[i, j]$ at time $t$ and $\sum_{i} \sum_{j} p_{i, j}(t)=1$. It is assumed that no reservation is possible: all passenger requests are expected to be fulfilled immediately. This can be considered to be the worst case (conservative) scenario, because if reservations were possible, this would always allow cars to be repositioned more efficiently. This is a significant difference from much of the previous work that includes reservations, making the system predictable in advance, and potentially more efficient.

Trip requests generated are assigned at each time step to available vehicles through the Kuhn-Munkres algorithm (Hungarian algorithm, [36]), which matches vehicles and requests to minimize total travel distance. A vehicle is considered to be available in any of the following cases:

- it is charging and has been charging for more than a minimum charging time (state 1$)$;

- it is parked idle (state 2);

- it is currently transporting a passenger (state 3)

In the last case, the vehicle will travel to pick up the next passenger after the current one has reached their destination. In terms of system states the constraint is: 


$$
{ }^{1} s_{v}+{ }^{2} s_{v}+{ }^{3} s_{v}=1
$$

A further necessary condition is that the vehicle has enough charge for the specific trip request:

$$
\left(w_{v}(t)+d_{k, i}+d_{i, j}\right) \cdot E C<\left(q_{v}(t)-q_{\min }\right) \cdot C A P
$$

Node $k$ is the last destination of the vehicle (or the current position for idle or charging vehicles), $w_{v}$ is the distance to the last destination, $E C$ is the energy consumption, $q$ is the SOC and $C A P$ is the battery capacity. Note that the charge available is calculated considering that vehicles should never be below a minimum SOC when the destination is reached, in order to have enough charge to move to a charging station. When a request is assigned, the vehicle's distance to destination $w_{v}$ is increased, the destination node $k$ is updated and the vehicle's state is changed:

$$
\begin{aligned}
& w_{v}(t+1)=w_{v}(t)+d_{k, i}+d_{i, j} \\
& { }^{1} s_{v}(t+1)=0 \\
& { }^{2} s_{v}(t+1)=0 \\
& { }^{3} s_{v}(t+1)=1
\end{aligned}
$$

At each time step, the distance to destination will decrease: $w_{v}(t+1)=$ $w_{v}(t)-u(t)$, until $w_{v}(t)=0$ and the destination is reached.

The request is rejected if it is not assigned to a vehicle in the same time step, thus not allowing request queuing. The number of these rejected requests is later used as one indicator of system performance. 


\subsection{Charging stations}

Vehicles can only charge their batteries at designated charging stations. The charging stations are placed at specific nodes in the grid, and vehicles need to travel to these nodes in order to charge. Autonomous vehicles are assumed to be able to connect to a charging pod automatically and with minimum delay. Several implementations of this technology have been proposed [37]. Charging station congestion has not been assessed in this work, so it is assumed that charging spaces are always available. This is considered reasonable since charging stations would be sized according to usage patterns.

A vehicle with no requests pending moves to a charging station either when its battery's SOC is below a certain level $q_{\text {charge }}$, or when it has not been assigned requests for a certain period of time (maximum idle time $\left.a_{i d l e}\right)$. In this last case, this also helps the re-positioning of vehicles that find themselves in peripheral nodes with limited request rates. Vehicle reaching a charging station start charging after a certain delay $a_{c o n}$, to account for the time to physically connect to the pod. Once it starts charging, the vehicle keeps charging for at least a minimum charging time $a_{\text {charge }}$ to avoid continuous disconnection (see Table 3). After the time threshold is reached, the vehicle becomes available for passengers, while still connected to the grid. It therefore participates in the assignment algorithm, although with lower priority compared to an idle vehicle: if the two vehicles have the same distance to the request, the idle vehicle is chosen. The vehicle stays connected otherwise and it therefore counts as an additional storage for the grid.

The number of time steps of delay in each case is assigned to the variable 
$a_{v}(t)$, which evolves as follows:

$$
\begin{aligned}
a_{v}(t+1) & =\max \left(a_{v}(t)-1\right. \\
& +\left({ }^{0} s_{v}(t+1) \cdot{ }^{5} s_{v}(t)\right) \cdot a_{\text {charge }} \\
& +\left({ }^{2} s_{v}(t+1)-{ }^{2} s_{v}(t)\right) \cdot a_{\text {idle }} \\
& \left.+\left({ }^{5} s_{v}(t+1) \cdot{ }^{4} s_{v}(t)\right) \cdot a_{\text {con }}, 0\right)
\end{aligned}
$$

The first two terms inside the max expression in (6) accounts for the decreasing delay at each time step. The third term accounts for the minimum charging time, the fourth term for the maximum idle time, and the fifth term for the time to connect to a charging station. Note that $a_{v}$ is always nonnegative. Moreover, when terms 3 and 5 are nonzero, they are always positive and $a_{v}(t)$ in (6) is necessarily zero per (12) and (17).

\subsection{Charging}

All vehicles in the simulation are battery electric and therefore need to charge to be able to serve the passenger requests. A heuristics-based charge scheduling algorithm is used in the simulations, and the interaction between the power grid and the vehicles is mediated by the electricity price from the grid. A price-based demand response is helpful in balancing the grid in the case of high penetration of renewable energy [38].

The charge scheduling algorithm is based on an 'agent price' $\pi_{v}$. In this work, the agent price is a measure of the value of electricity stored in each vehicle. In other words, the agent price is the 'perceived' value of electricity for each agent (vehicle) at a certain time. 
The vehicle will buy electricity when the agent price is higher than the electricity price. In particular, the vehicle will charge at a rate proportional to the difference between $\pi$ and the electricity price, up to a maximum $\Delta_{\max }$, which corresponds to the maximum charging rate $p_{\max }$. The unconstrained rate of charge, or the proportion of the maximum power that would be used in absence of other constraints, can therefore be defined as:

$$
b_{v}(t)=\max \left(\min \left(\frac{\pi_{v}(t)-m(t)}{\Delta_{\max }} \quad, \quad 1\right) \quad, \quad-1\right)
$$

Where $m(t)$ is the price of electricity from the grid. $\Delta_{\max }$ was set at $30 \mathrm{JPY} / \mathrm{kWh}$. This behavior was introduced to increase the system stability when the agent price is close to the price of electricity. The energy exchanged (in $\mathrm{kWh}$ ) at time step $t$ is therefore:

$$
\begin{array}{r}
e_{v}(t)=\left({ }^{0} s_{v}(t)+{ }^{1} s_{v}(t)\right) \cdot \max \left(\operatorname { m i n } \left(b_{v}(t) \cdot \frac{p_{\max } \cdot \ell}{60},\right.\right. \\
\left.\left.C A P \cdot\left(q_{\max }-q_{v}(t)\right)^{+}\right), C A P \cdot\left(q_{\min }-q_{v}(t)\right)^{-}\right)
\end{array}
$$

The shorthand notation used in (8) is defined as: $x^{+}:=\max (x, 0), x^{-}:=$ $\min (x, 0)$. Equation (8) refers to the energy reaching the vehicle and thus does not account for the efficiency of the battery, which is counted only for the charging cycle when calculating the cost of the energy. $e_{v}(t)$ is positive when the vehicle is charging, and negative when discharging.

It is assumed that in order to calculate the agent price, predictions of short-term future price and transport demand are available to the system up to a certain horizon. Moreover, it is assumed that the electricity price is not 
influenced by the behavior of SAEVs (the model simulates a small enough fleet of SAEVs). This is justified by the scale used in the simulations. Even assuming 2000 vehicles (the maximum number used in simulations) charging at the same time at $10 \mathrm{~kW}$, the total load would be $20 \mathrm{MW}$, which is less than a thousandth of the average power generation by Tokyo Electric Power Company (TEPCO) of $\sim 23 \mathrm{GW}$ in 2015 [39].

The algorithm is based on the average of expected future prices and transport demand as follows:

$$
\begin{gathered}
\pi_{v}(t)=\frac{\sum_{j=1}^{h z}\left(m(t+j)+\lambda^{\prime}(t+j) \cdot P S P / E C\right)}{2 \cdot q_{v}(t) \cdot h z} \\
\lambda^{\prime}(t)=\frac{\lambda(t) \cdot 60}{\ell \cdot \sum_{t-1440 / \ell}^{t-1} \lambda(t)}
\end{gathered}
$$

Where $\pi_{t}$ is the agent price at time $t, h z$ is the prediction horizon for future prices, $P S P$ is the passenger service priority factor, and $\lambda^{\prime}$ represents the relative rate of requests in the time step compared to the total in the previous 24 hours, normalized as a rate of trips per hour. The PSP (expressed in $\mathrm{JPY} / \mathrm{km}$ or $\mathrm{USD} / \mathrm{km}$ ) is a weighting parameter used to allocate a certain amount of energy to transport requests as opposed to energy storage. A higher PSP would put more priority on passengers, reducing probability of dropped requests and possibly waiting times, but also rendering storage less effective. The simple equation allows for a fast calculation of the agent price at each time step for each vehicle.

The state of charge of each vehicle will then evolve according to:

$$
q_{v}(t+1)=q_{v}(t)+\frac{e_{v}(t)}{C A P}-\frac{u(t) \cdot E C}{C A P} \cdot\left({ }^{3} s_{v}(t)+{ }^{4} s_{v}(t)\right)
$$


In accordance with the model described, the state variables evolve according to the following equations:

$$
\begin{gathered}
{ }^{0} s_{v}(t+1)={ }^{0} s_{v}(t)+\left({ }^{5} s_{v}(t)-{ }^{0} s_{v}(t)\right) \cdot\left(1-\operatorname{sgn}\left(a_{v}(t)\right)\right) \\
{ }^{1} s_{v}(t+1)={ }^{1} s_{v}(t)+{ }^{0} s_{v}(t) \cdot\left(1-\operatorname{sgn}\left(a_{v}(t)\right)\right) \\
{ }^{2} s_{v}(t+1)={ }^{2} s_{v}(t)+{ }^{3} s_{v}(t) \cdot\left(1-\operatorname{sgn}\left(w_{v}(t)\right)\right) \\
{ }^{3} s_{v}(t+1)={ }^{3} s_{v}(t) \cdot \operatorname{sgn}\left(w_{v}(t)\right) \\
\text { and } \left.-1)={ }^{4} s_{v}(t)-{ }^{4} s_{v}(t)\right) \cdot\left(1-\operatorname{sgn}\left(w_{v}(t)\right)\right) \\
{ }^{5} s_{v}(t+1)={ }^{5} s_{v}(t)-{ }^{5} s_{v}(t) \cdot\left(1-\operatorname{sgn}\left(a_{v}(t)\right)\right)+{ }^{4} s_{v}(t) \cdot\left(1-\operatorname{sgn}\left(w_{v}(t)\right)\right) \\
\text { with sgn }(x) \text { the sign function which is } 0 \text { when } x=0,1 \text { when } x \text { is positive }
\end{gathered}
$$

\subsection{Operating reserve model}

The potential for the cars to act as operating reserve is subject to the speed at which they can deploy capacity, and for how long. To evaluate these factors, a request mechanism was implemented in the model. An operating reserve request in this model is characterized by a duration $\Gamma$ and an allowed delay $\delta$. At time step $t$ of the simulation the system is tested to calculate 
the maximum theoretical operating reserve power available $r_{\max }(t)$. This is the maximum constant power deliverable for the request duration.

It is assumed that during an operating reserve request the vehicles will put priority in satisfying the grid operator request over new passengers' requests if necessary. Vehicles can therefore be called back to charging stations to contribute. The rate at which these vehicles can be called back determines the delay in fulfilling the request and the overall energy and power available. Vehicles with pending passenger requests (already accepted) can move to charging stations only after transporting their passengers, thus affecting their connection time or grid waiting time. Moreover, in these special cases, vehicles are allowed to reach the full range of battery capacity from $0 \%$ to $100 \%$ SOC if needed.

The calculation of the power available progresses with a time step $\tau$ through the duration of the grid operator request. For each vehicle $v$, the time needed to deploy capacity $\alpha$ is calculated, subtracting the allowed delay $\delta$ of the request:

$$
\begin{aligned}
\alpha_{v} & =\max \left(0, \quad\left({ }^{2} s_{v}(t)+{ }^{3} s_{v}(t)+{ }^{4} s_{v}(t)\right) \cdot\left(\frac{w_{v}(t)+d_{i, j}}{u(t)}+a_{c o n}\right)\right. \\
& \left.+{ }^{5} s_{v}(t) \cdot a_{v}(t)-\delta\right)
\end{aligned}
$$

with $i$ the vehicle's node (or current passenger's drop-off node) and $j$ the node with the closest charging station. Vehicles which are already connected to a charging station will always have a time delay of 0 minutes. The state of charge at the time of connection to the charging station is also calculated for each vehicle: 


$$
q_{v, \text { conn }}=q_{v}(t)-\left(w_{v}(t)+d_{i, j}\right) \cdot \frac{E C}{C A P} \cdot\left({ }^{2} s_{v}+{ }^{3} s_{v}+{ }^{4} s_{v}\right)
$$

The energy delivered at each time step of the request by each vehicle is referred to with $\epsilon_{v}(\tau)$. In order to have the maximum energy delivered the following objective function should be maximized:

$$
f_{p}\left(\epsilon_{v}\right)=\sum_{\tau} \sum_{v} \epsilon_{v}(\tau)
$$

Also, it is desirable that the minimum number of vehicles are used for the grid request, in order to limit the disruption to the transport service. The cost function can therefore be introduced:

$$
\begin{gathered}
f_{s}\left(c_{v}\right)=\cdot \sum_{v} c_{v} \\
c_{v} \in\{0,1\}
\end{gathered}
$$

where $c_{v}$ is a binary variable that represents the commitment of vehicle $v$ for the request. Equation (21) can be considered the cost of commitment of each vehicle, which is paid only when the vehicle is used (called back to the charging station). The overall objective function to maximize is then:

$$
f(x)=f_{p}\left(\epsilon_{v}\right)-b \cdot f_{s}\left(c_{v}\right)
$$

$b$ is the secondary objective's relative weight. Only vehicles that are connected at time $\tau$ can contribute to energy delivery:

$$
\epsilon_{v}(\tau)=0 \quad \tau<\alpha_{v}
$$


moreover, the energy deliverable at each time step is constrained by the maximum power:

$$
0 \leqslant \epsilon_{v}(\tau) \leqslant \frac{p_{\text {peak }}}{60}
$$

The state of charge of each vehicle has to remain within the interval $[0,1]$ at each time step:

$$
0 \leqslant q_{v, \text { conn }}-\frac{\sum_{k=1}^{\tau} \epsilon_{v}(k)}{C A P} \leqslant 1 \quad \tau \in \Gamma
$$

The commitment costs are introduced as:

$$
\sum_{\tau} \epsilon_{v}(\tau) \leqslant \frac{p_{p e a k}}{60} \cdot \Gamma \cdot c_{v}
$$

meaning that if the commitment variable $c_{v}$ is 0 , vehicle $v$ can not supply energy. A limit to how many vehicles can be used was also introduced, to ensure that there are always enough vehicles left for the transport service:

$$
\sum_{\tau} c_{v} \leqslant z \cdot V
$$

where $z$ is the maximum ratio of vehicles that can be used for the grid request. This value is only relevant during periods of relatively low passenger request rates, for example at night, since most of the times the number of vehicles used is limited by other factors. In the simulations, $z$ was chosen as 0.7. Choosing a lower value would put more priority on the passenger requests. The last constraint dictates that the power delivered should stay constant during the request time: 


$$
\sum_{v} \epsilon_{v}(\tau+1)=\sum_{v} \epsilon_{v}(\tau) \quad \tau \in \Gamma
$$

The problem can therefore be stated as:

$$
\begin{array}{ll}
\underset{x}{\operatorname{maximize}} & f(x) \\
\text { subject to } & (22),(24),(25),(26),(27),(29)
\end{array}
$$

The problem (30) is a mixed integer linear program and was solved with the built-in MATLAB function intlinprog.

The disruption to the transport service during and after the request is also investigated. During a request, the vehicles participating will stay in state 0 , thus not available, and their power exchange will be determined by the optimization results. Vehicles not participating in the operating reserve request are not permitted to exchange power with the grid during the request time, so that the system acts as a single agent. Moreover, as mentioned previously, during the request vehicles can discharge until they reach 0 state of charge. The final impact on the transport service is assessed by the number of extra rejected requests and extra minutes of waiting times when compared to the base scenario.

\subsection{Transport system evaluation}

In order to evaluate the economic feasibility of the SAEV system, a conservative estimate of the costs was made (see Table 4). The values were estimated based on currently available electric vehicles and on estimates of the price of autonomous vehicle control hardware (see section 3.9). The life expectancy of the vehicle is defined in years, while the life of the battery is 
defined by its total number of full cycles (100-0-100\% SOC). The cost of the vehicle will therefore be considered a fixed cost based on the lifetime of the vehicle, while the cost of the battery will depend on its use (charge/discharge cycles).

To assess the cost of the system, the break-even price (BEP) is used. This is defined as the minimum price per $\mathrm{km}$ the passengers have to pay to cover the total costs of the SAEV system. Any price higher than the BEP will be a net profit for the system. The BEP is calculated based on the results of the simulation:

$$
B E P=\frac{\sum_{v}\left(V C_{v}+F C_{v}\right)}{\sum_{v} W_{p a s s, v}}
$$

where $V C_{v}$ and $F C_{v}$ are the variable and fixed cost, respectively, for vehicle $v$, and $W_{\text {pass }, v}$ is the distance traveled with passengers for vehicle $v$. The BEP is expressed in JPY $/ \mathrm{km}$ (or USD $/ \mathrm{km}$ ). The system would incur further overhead costs, such as the cost for managing the assignment system. However, for simplicity, these are assumed to be included in the overall price of the vehicle (fixed costs). The variable and fixed costs are:

$$
\begin{gathered}
V C_{v}=\frac{\sum_{t} e_{v}(t)}{L_{\text {battery }} \cdot C A P} \cdot C_{\text {battery }}+\sum_{T} e_{v}(t) \cdot m(t) \\
F C_{v}=\frac{C_{c a r} \cdot T \cdot \ell}{L_{c a r} \cdot 525600}
\end{gathered}
$$

Where $C_{\text {battery }}$ and $C_{c a r}$ are the cost of the battery and the vehicle, respectively; $L_{\text {battery }}$ and $L_{\text {car }}$ are, respectively, the life of the battery (in number of cycles) and the vehicle (in years); 525600 is the total number of minutes in a year. 
The variable cost depends on the specific vehicle (function of the total distance traveled), while the fixed cost is the same for each vehicle. The total costs are then the sum of all the individual vehicles' costs. Moreover, the system efficiency is also calculated as:

$$
\text { Efficiency }=\frac{\sum_{v} W_{\text {pass }, v}}{\sum_{v} W_{t o t, v}}
$$

where $W_{t o t, v}$ is the total distance traveled.

\subsection{Model assumptions, validation and limits}

Validation of the model is important in ensuring the credibility of the results. While it is not possible to validate the model with real world demonstration or experiment, it is possible to validate the internal consistency and performance against theoretical expectations. The influence of model parameters was verified with sensitivity analysis. Model parameters such as idle time and battery capacity were chosen through sensitivity analysis to maximize BEP and minimize waiting times. The model's limitations come primarily from the assumptions made to make the simulations possible with limited data. The main simplifying assumption is related to the transport simulation, as the city's road network is not considered. This is to allow for a faster simulation time and also due to the difficulty of properly considering traffic congestion in the simulations.

Other simplifying assumptions are related to the energy aspects of the model. Detailed charge and discharge behavior of batteries is not considered: charge power and charging/discharging efficiency are assumed to be the same at any SOC level. This is considered acceptable for this level of analysis. 
This is further justified by the fact that the simulations assume relatively conservative charging power levels. Moreover, the detailed electric grid is not considered: the model assumes that there are no transmission capacity constraints, and does not consider transmission losses. This is justified by the assumption that the centralized nature of charging stations allow vehicles to be connected directly to the medium or high voltage transmission grid, as opposed to the distribution network. Moreover, the positioning of these charging stations could be optimized to minimize power losses. These aspects were not considered in this paper, and are planned as the focus of future work.

Another limitation of the model is the consideration of static transport patterns. It is possible that the service will also attract people currently using public transportation or other means, thus affecting the position and time of trips from the survey. All these simplifying assumptions have been previously used in most of the models reviewed in section 2. Numerical assumptions in the case study were backed by references whenever possible. The results are intrinsically uncertain due to the lack of real-world examples of commercial shared autonomous vehicle fleets and due to the experimental nature of this technology, which makes it impossible to compare the model with real data. However, despite this limitation, the internal and theoretical consistency should provide sufficient validation of the model's demonstrative analytical capacity for the case study presented.

\subsection{Case study: transport data}

The case study examined here is based on the Tokyo Person Trip Survey 2008 [40], a survey of around 2 million trips in the Tokyo metropolitan area. The 2008 survey is the latest available survey released for Tokyo. Although 
somewhat old, the demographics and infrastructure of Tokyo has remained stable and it is expected that this importantly implies a relatively stable demand pattern when compared to 2008. Infrastructure and ridership of all major railways are shown to be mostly unchanged between 2008 and 2015, and in the same period, the length of roads in Tokyo city changed by less than $1 \%[41]$.

The survey associates the origins and destinations of trips to zones, corresponding to specific addresses in Tokyo. These geographical zones were used in the model as the reference nodes. The geographic coordinates of the zones were found from the addresses reported in the survey using the Google Maps Geocoding API [42]. The approximate area of the zones was also found and was used as the area associated with the node. In order to have a representative collection of trip characteristics for a city, the area of service in the simulations was limited to a central 40x40 km area of Tokyo, so only trips starting and ending in zones in this area were considered. This is approximately equivalent to the 23 special wards of Tokyo, which are the core and the most populous part of the city. The selected area includes 514 zones, which are selected as the nodes in the model. $34.5 \%$ of all the trips in the survey start and end in the central zones selected. The centers of the nodes selected are shown in Fig. 2.

Most of the trips in the survey are by public transport. However, for the purpose of this study, only trips by car or taxi were considered. These are the trips with characteristics more likely to be similar to trips done with the SAEV system. These trips represent about $20 \%$ of the total trips in the survey. Trips by car or taxi in the selected area are a total of 73,000 , or 


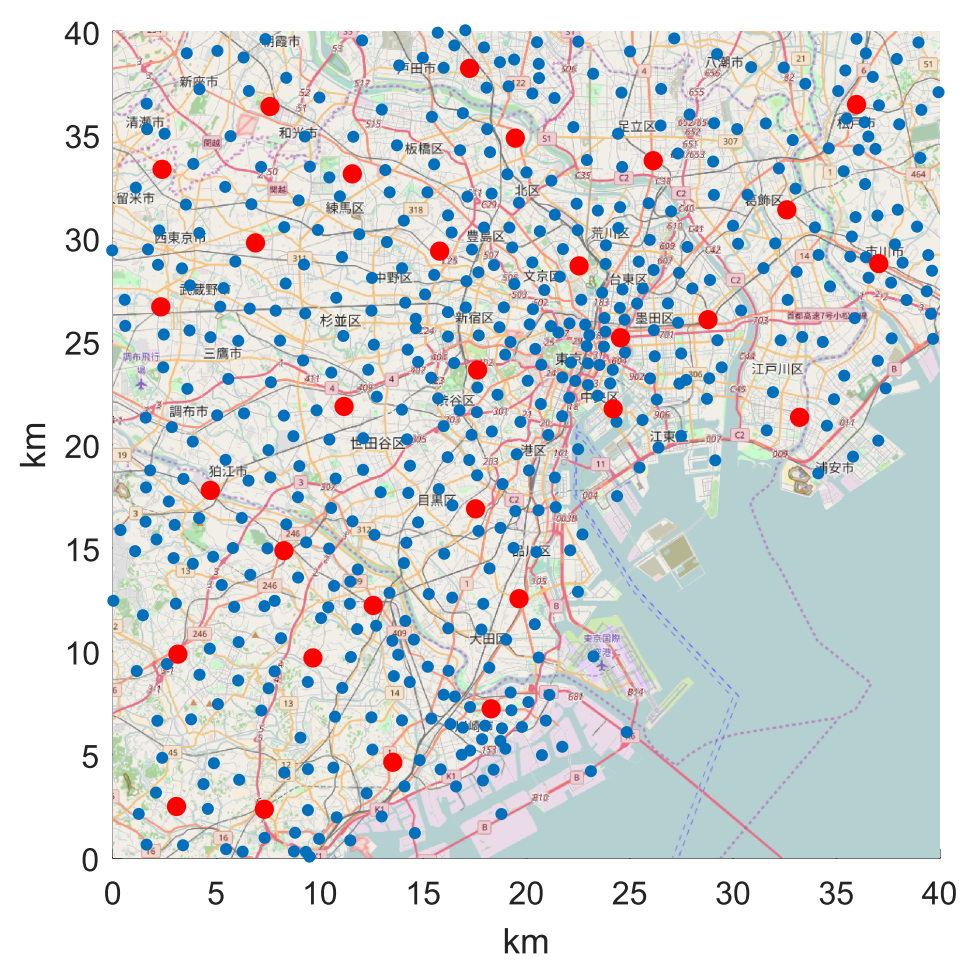

Figure 2: Map of nodes in central Tokyo. Blue and red dots-Dots represent the approximate location of the center of the node. From [40]. Red dots represent the position of nodes with charging stations in the simulation.

$3.8 \%$ of the total trips in the survey. Of these, only about 70,000 are found to have a reported trip starting time. These are the trips that were used in this work. Although this is a small proportion of the total trips, it should be noted that the aim of the system is not to cover $100 \%$ of trips, but rather enough of them for the system to be sustainable. Further work could consider the migration of bus or train-based trips onto the SAEV service, but that is not the focus of the current study.

Each trip in the survey also has a starting time and a weight. The 


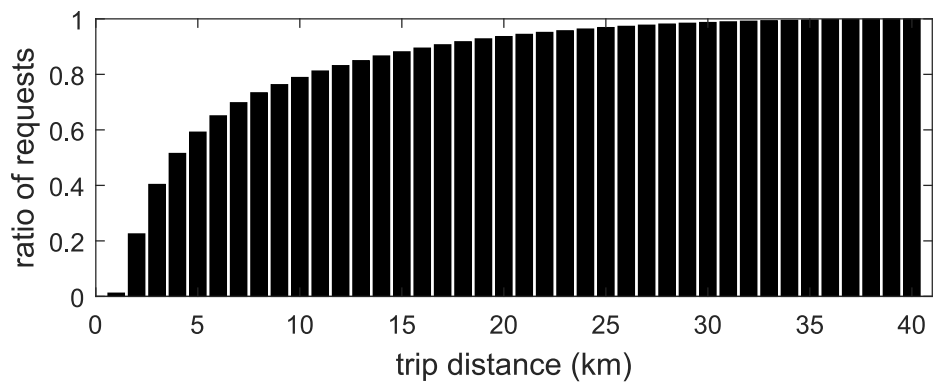

(a)

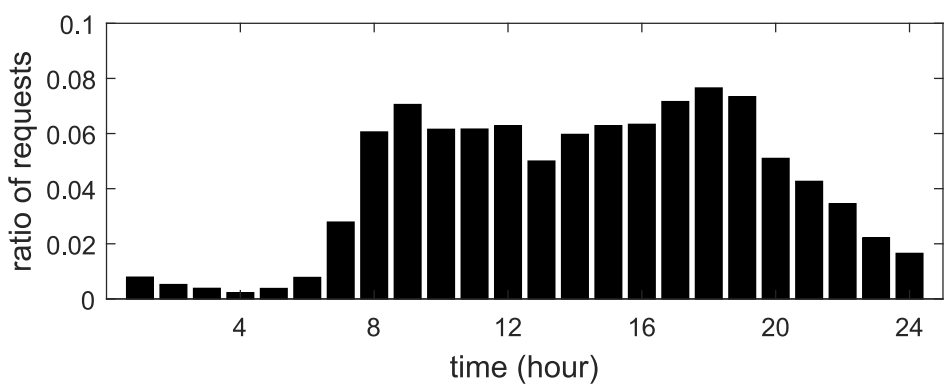

(b)

Figure 3: (a) cumulative distribution of trip distance and (b) distribution of trip starting time from transportation survey [40]

weight is used to indicate the relative significance of that specific trip and to normalize the survey results over the total demographics of Tokyo. Although trip starting time is specified by hour and minute, it was found that most trips start at minute 0 of each hour. The minute information was therefore judged to be unreliable and was not considered in this work.

Each selected trip $k$ is then defined with four values $\left[r w_{k}, r t_{k}, r o_{k}, r d_{k}\right]$ to indicate respectively associated weight, hour of departure (0-23), origin node, and destination node. The probability $p_{i, j}(t)$ was found as: 


$$
p_{i, j}(t)=\frac{\sum_{k^{\prime}} r w_{k^{\prime}}}{\sum_{k} r w_{k}}, \quad k: r t_{k}=t^{\prime}, k^{\prime}: r t_{k^{\prime}}=t^{\prime}, r o_{k^{\prime}}=i, r d_{k^{\prime}}=j
$$

where $t^{\prime}=\lfloor((t \cdot \ell-1) \bmod 1440) / 60\rfloor$ is the hour of the day corresponding to time step $t$.

The relative number of trips departing at hour $h$ from the survey is:

$$
f(h)=\frac{\sum_{k^{\prime}} r w_{k^{\prime}}}{\sum_{k} r w_{k}}, \quad k^{\prime}: r t_{k^{\prime}}=h
$$

At each time step $t$ (at hour of the day $h$ ), the rate of the Poisson process in $(2)$ is then:

$$
\lambda(t)=T P H \cdot 24 \cdot f\left(t^{\prime}\right) \cdot \ell / 60
$$

where $T P H$ is the average rate of trips per hour.

The ratio of trip distance to Euclidean distance (tortuosity factor) was determined by testing random trips within the selected area and averaging the value of effective distance to Euclidean distance between the coordinates. Only coordinates associated with an address in the immediate vicinity were considered, thus excluding unoccupied areas. The associated addresses and the actual travel distance was found with the Google Maps Geocoding API [42]. 1298 origin-destination pairs were tested, with a resulting average $\beta=$ 1.48 , which was used in the simulations. It was also confirmed that the value of $\beta$ is not significantly correlated with any trip characteristic, such as travel distance or geographic area, so that the use of a single average value can be considered acceptable. 
30 charging stations were positioned in total. A sensitivity analysis showed that increasing the number of stations up to 30 had a significant impact on the model, while a higher number of charging stations did not significantly change the results. The position of charging stations in the case study was determined in order to minimize (to a large extent) the distance of travel from each node to the closest station. The first station was positioned in the node for which the sum of the distances to all other nodes is minimal. Subsequent stations were positioned in the same way, taking into account the presence of previous stations (i.e. considering only distances to the closest station). This algorithm is not optimal, but provides a good approximation of a distribution minimizing travel distances from each point.

Three levels of traffic congestion were considered: peak, average, and offpeak, at 20, 30 and $40 \mathrm{~km} / \mathrm{h}$, respectively. The lowest speed of $20 \mathrm{~km} / \mathrm{h}$ was chosen as the reported average speed in central Tokyo at peak time [43]. The average and off-peak speeds were chosen as 1.5 and 2 times the peak speed, as precise data on average speed in Tokyo could not be found. It should be noted that the off-peak speed does not affect results significantly, since the limiting factor for fleet sizing and operating reserve service is the minimum speed at the moment of maximum transport request rate. The speed in $\mathrm{km} / \mathrm{h}$ is related to the distance traveled in one time step $u(t)$ in the simulation as: $u(t)=\operatorname{speed} \cdot \ell / 60$.

\subsection{Case study: vehicle characteristics and costs}

The parameters chosen for the vehicles are summarized in Table 4. Electricity consumption of vehicles $E C$ was chosen at $0.15 \mathrm{kWh} / \mathrm{km}$, taken from [44] and similar to the Nissan Leaf energy consumption at city speeds 


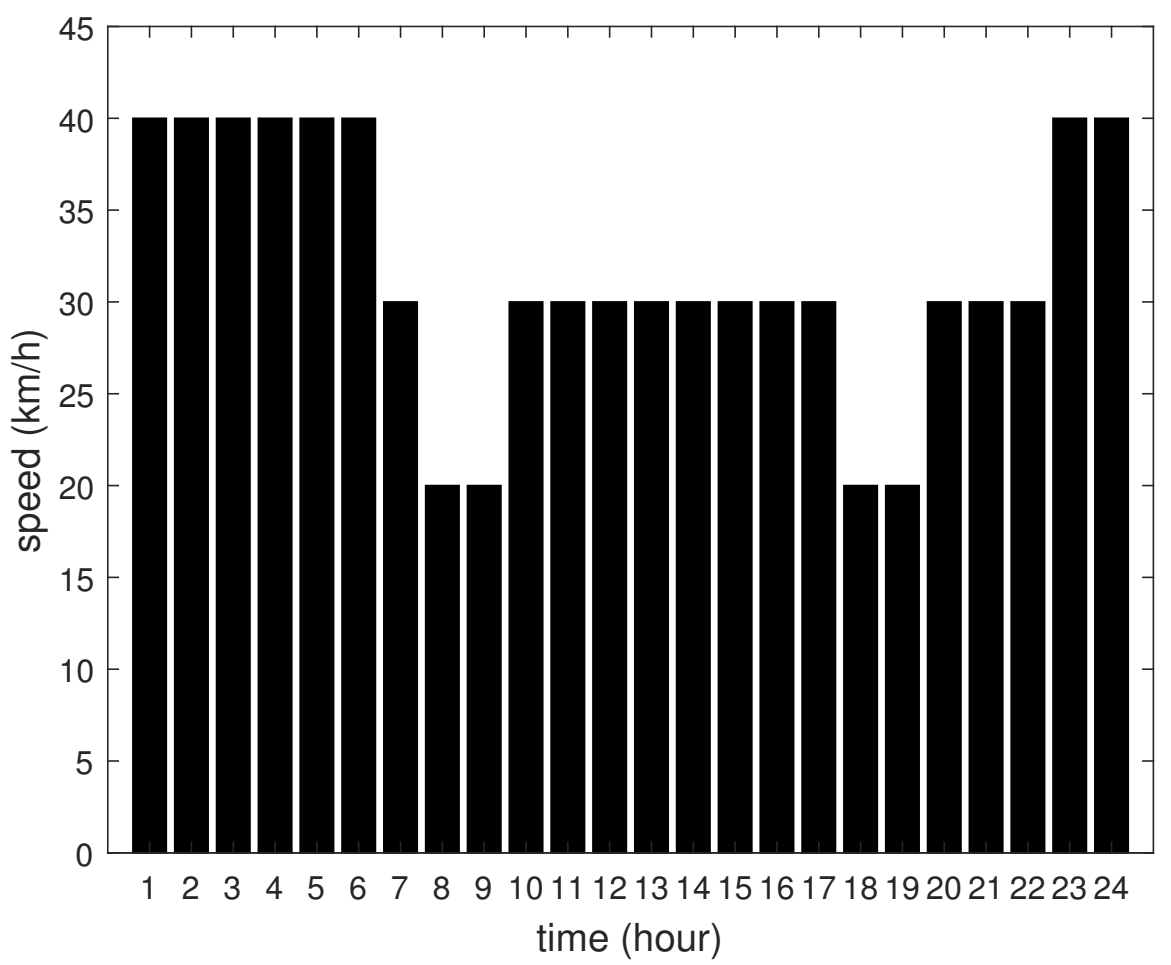

Figure 4: Average speed during the day.

[45]. Battery cycle life was estimated at 1500 full cycles. Studies show that lithium-ion batteries for electric vehicles have low capacity fade even after 1000 cycles [46]. Real-life examples for lithium-ion batteries confirm these findings. Tesla Model S batteries have shown less than $10 \%$ capacity degradation over 700 cycles in surveys of private users [47] and the Powerwall (stationary Li-ion battery) from the same company offers a warranty of $60 \%$ capacity retention after 10 years with unlimited cycles [48]. A more controlled charging schedule can also contribute to increase battery life. Uddin et al. demonstrate an increase in battery life for electric vehicles when using smart 
charging with V2G compared to private charging at home [49]. Capacity fade of the battery is not considered in the model, thus the stated capacity should be considered as an average during the battery life.

Prices are given in Japanese Yen (JPY) and US Dollars (USD), with a conversion rate of 1 JPY $=0.009$ USD (rate as of January 2018). Autonomous driving technology is expected to add $\$ 7,000$ to $\$ 10,000$ to the price of a vehicle by 2025 [50]. Fagnant and Kockelman estimate $\$ 10,000$ added cost for early adoption [51]. A conservative estimate of 5 million JPY $(\$ 45,000)$ was used for the cost of the vehicle and other expenses (such as control center, maintenance etc.), excluding battery. The cost of the battery was calculated assuming $200 \mathrm{USD} / \mathrm{kWh}(22,200 \mathrm{JPY} / \mathrm{kWh})$ [52].

Maximum charging/discharging power was set at $10 \mathrm{~kW}$ for normal operation and at $20 \mathrm{~kW}$ for short times (peak power when responding to grid requests). These power levels can be provided by several existing technological standards [34], which are not discussed in this work. Simulations were also run with a hypothetical $50 \mathrm{~kW}$ connection to investigate the influence of charging speed on performance.

Minimum SOC was set at $25 \%$ in order to enhance durability of the battery (see Table 3) [53]. The maximum SOC was set at $80 \%$ in normal operation, to increase the ability to absorb excess generation from the grid when needed. This has also been shown to further extend battery life as lithium-ion batteries suffer higher stress at high SOC [54].

The vehicle connection time time needed for the vehicle to connect to a charging station has been assumed to be 3 minutes. This includes the time to park the vehicle and connect it to a charging pod. Internal parameters 
Table 3: Summary of simulation parameters

\begin{tabular}{lll} 
Name/symbol & Value & Unit \\
\hline$q_{\text {charge }}$ & 0.35 & - \\
$q_{\text {min }}$ & 0.25 & - \\
$q_{\text {max }}$ & 0.8 & - \\
$\ell$ & 1 & minutes \\
$a_{\text {con }}$ & 3 & minutes \\
$a_{\text {charge }}$ & 30 & minutes \\
$a_{\text {idle }}$ & 5 & minutes
\end{tabular}

of the model, such as minimum charging time and maximum idle time, were chosen through sensitivity analysis to minimize waiting times and BEP.

Due to the uncertainties related to future implementations of autonomous driving technology, conservative parameters were chosen in the simulations. However, it is possible that prices and vehicle performance would be better than in the current study. For example, cheaper and more durable batteries could increase the energy available and the storage capability, while keeping costs low.

\subsection{Case study: electricity prices}

The influence of electricity pricing was also studied. Three example price profiles were considered to test the model (see Fig. 5):

1. TOD - Time-of-day pricing with 2 price periods (peak/off-peak).

2. TOD+solar - TOD with high solar penetration, with peaks at early morning and evening. 
Table 4: Summary of technical and economic assumptions

\begin{tabular}{lll} 
Name/symbol & Value & Unit \\
\hline$E C$ & 0.15 & $\mathrm{kWh} / \mathrm{km}$ \\
$C A P$ & 50 & $\mathrm{kWh}$ \\
$\eta$ & 0.9 & - \\
$p_{\text {max }}$ & 10 & $\mathrm{~kW}$ \\
$p_{\text {peak }}$ & 20 & $\mathrm{~kW}$ \\
$L_{\text {car }}$ & 5 & years \\
$L_{\text {battery }}$ & 1500 & full cycles \\
$C_{\text {car }}$ & $5,000,000$ & JPY \\
& 45,000 & USD \\
$C_{\text {battery }}$ & $1,110,000$ & JPY \\
& 10,000 & USD
\end{tabular}

3. HighRe - Free-Wholesale electricity market with high renewable energy penetration: random price profile extracted from a gamma distribution with shape parameter $k=2$ and scale parameter $\theta=20$.

The electricity market in Japan is undergoing a process of liberalization, and some electricity providers already offer several time-of-day pricing options [55]. All price profiles were normalized to the same average value of $40 \mathrm{JPY} / \mathrm{kWh}(0.36 \mathrm{USD} / \mathrm{kWh})$, in order to investigate the ability of the proposed charge scheduling algorithm to minimize energy expenditures. The average price is conservatively higher than current average prices (about 20 to $30 \mathrm{JPY} / \mathrm{kWh}$ for TEPCO, depending on type of connection [55]) to account for a possible future rise of energy prices. This may happen due 


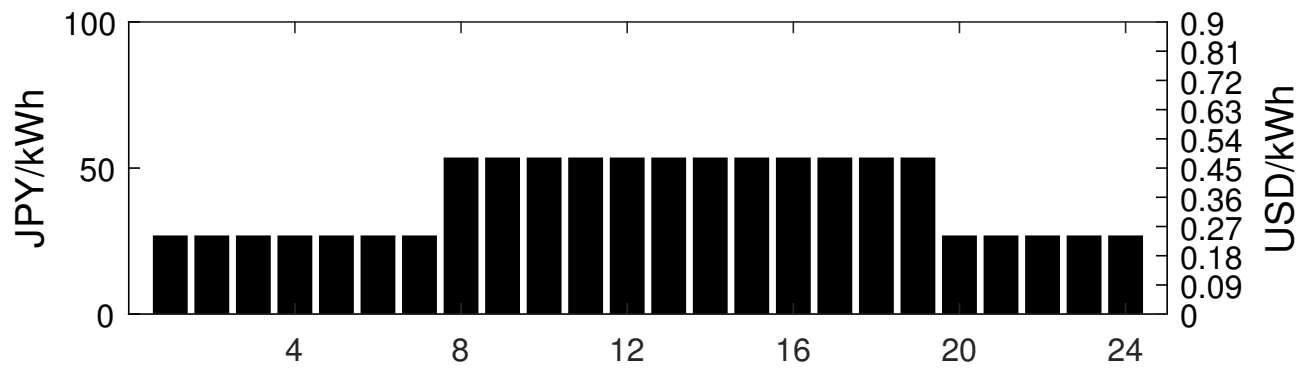

(a)

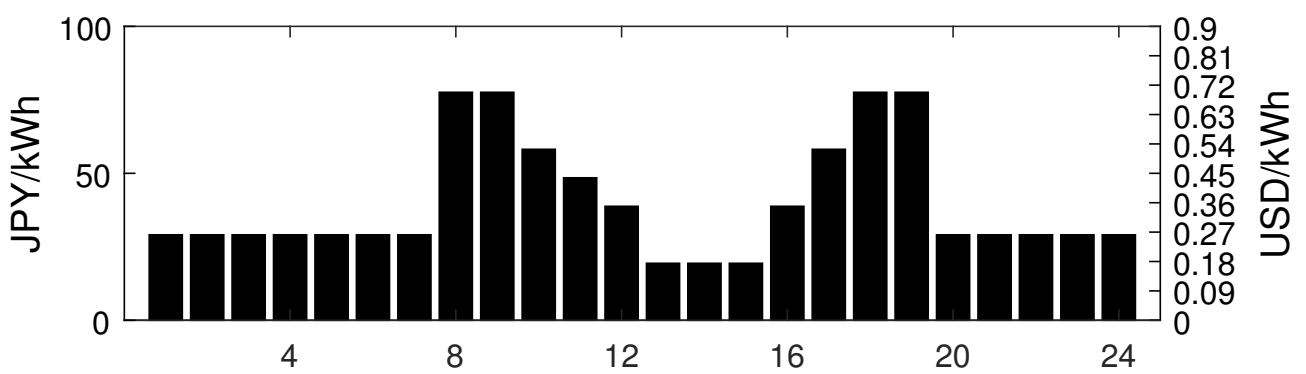

(b)

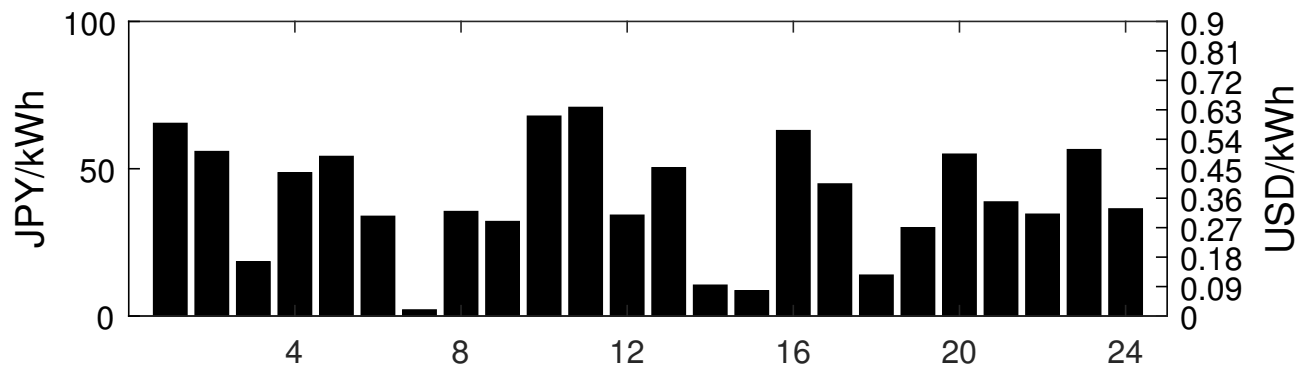

(c)

Figure 5: Comparison of 24-hours samples of price profiles used in the simulations. The average for all profiles is $40 \mathrm{JPY} / \mathrm{kWh}(0.36 \mathrm{USD} / \mathrm{kWh})$. (a) Time-ofday pricing with two periods; (b) time-of-day pricing with high solar penetration; (c) free market with high renewable energy penetration, modeled with a gamma distribution. 
to the rising cost of renewable energy subsidies and dispatch and cost of energy imports. In the third case, the system buys and sell electricity in the wholesale electricity market as a price taker. Although the generated price profiles in this case may not reflect the specific time distribution of prices in current markets, the temporal variability is justified by the high intermittent renewable energy penetration that is assumed in this scenario.

\section{Results from case study}

In this section, the results from the case study are presented. In the first section, the model is tested with different trip rates and number of vehicles to investigate the optimal fleet size as a trade-off between costs and transport service performance. In the second section, the effect of different price profiles on costs and transport performance is investigated using the optimal fleet size from the previous section. In the third section, the results of operating reserve simulations are presented for several request characteristics.

\subsection{Fleet sizing}

The aim of the first part of the simulations is to estimate the number of vehicles needed to satisfy the transportation requests. Simulations were run with different numbers of vehicles to estimate the optimal fleet size based on a number of output parameters. The parameters chosen were the waiting time for passengers, the break-even price of the system and the number of rejected requests.

The simulations have a time step of 1 minute over a period of 20 days. One extra day was added at the beginning to avoid start-up transients. The forecast horizon was set at 12 hours (720 time steps). Considering 
that both electricity price and passenger request rates change hourly, there are effectively only 12 distinct forecast values for each. Several trip rates were tested to show the influence of the rate on the system performance. This is useful to understand the sustainability of the system at different levels of request density, for example during the initial implementation phase when adoption rates are low. The passenger priority factor (PSP) was set at $100 \mathrm{JPY} / \mathrm{km}(0.9 \mathrm{USD} / \mathrm{km})$. This value implies a bigger priority for transport service compared to grid services, as it corresponds to an equivalent electricity price of $667 \mathrm{JPY} / \mathrm{kWh}$ (6 USD/kWh), considering the electricity consumption of vehicles.

The simulations are based on numbers of vehicles that are constant proportions of the average rate of trips in order to compare the results. For example, if the average rate of trips per hour is 500, then a proportion of 1.4 vehicles per average rate of trip per hour would be a fleet of $500 \cdot 1.4=700$ vehicles. The charging of the vehicles is managed by the charging algorithm introduced in section 3.4. A simple time-of-day hourly price profile with two periods was chosen for the electricity price (Fig. 5a), with an average electricity price of $40 \mathrm{JPY} / \mathrm{kWh}(0.36 \mathrm{USD} / \mathrm{kWh})$.

Results are shown in Fig. 6 and 7. Waiting times tend to stabilize when the number of SAEVs available is more than 1.2-1.4 times the average rate of trips per hour. The median waiting time drops to 7 minutes and $95 \%$ of requests are fulfilled within 18 minutes for a simulation with $1000 \mathrm{TPH}$ and 1400 vehicles (Fig. 6). Waiting times tend to reach a plateau with a certain amount of vehicles over TPH, depending on the TPH. After this plateau is reached, the waiting times are essentially not dependent on the number 


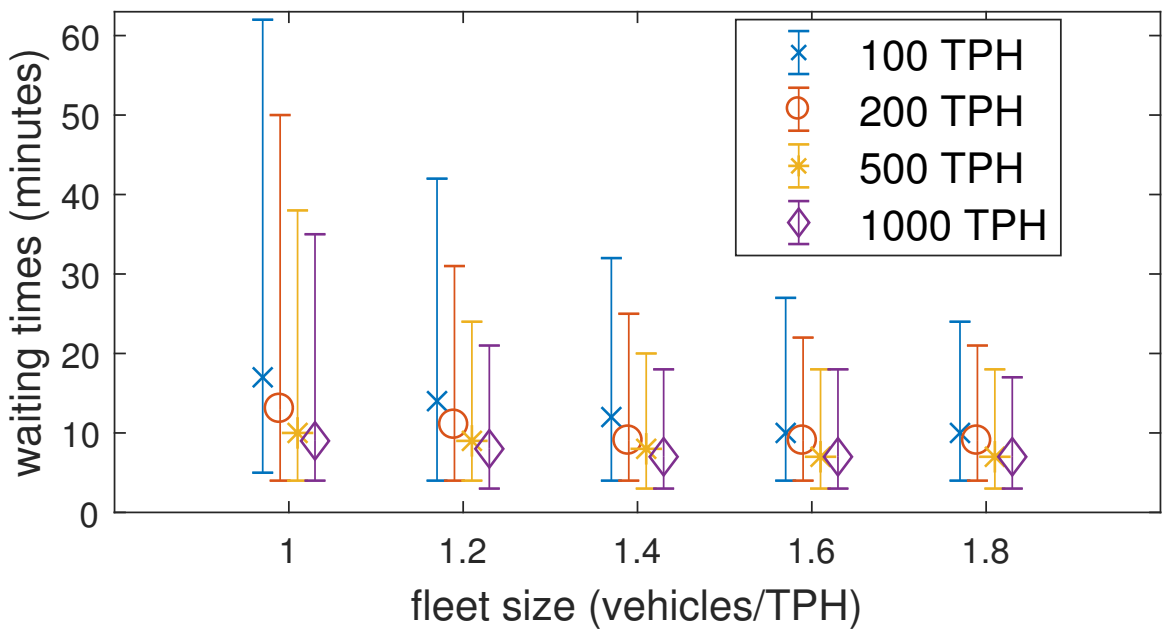

Figure 6: Waiting times as a function of the number of SAEVs and trips per hour (tph). The marker indicates the median value and the error bars the 5th and 95th percentiles.

of vehicles. Waiting times are strongly dependent on position and time of day, with increased waiting for requests in peripheral areas and during peak demand. An important characteristic of the system is that the expected waiting time is always known when a trip request is accepted (otherwise the request is rejected), so in a real case scenario the user can always plan in advance for the time needed (or use another means of transportation). Moreover, waiting times are quite predictable, depending on a certain time and position of the trip request. All these factors may be investigated further in future work.

Break-even prices tend to increase as the fleet size increases (Fig. 7a). This is due to the increased investment needed for a larger fleet while efficiency levels and number of passengers served are stable (no rejected re- 


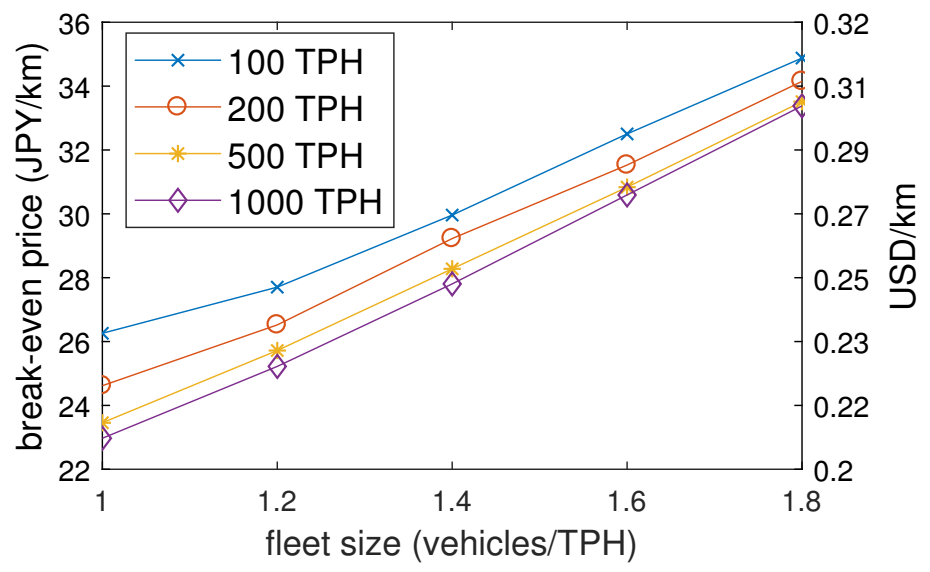

(a)

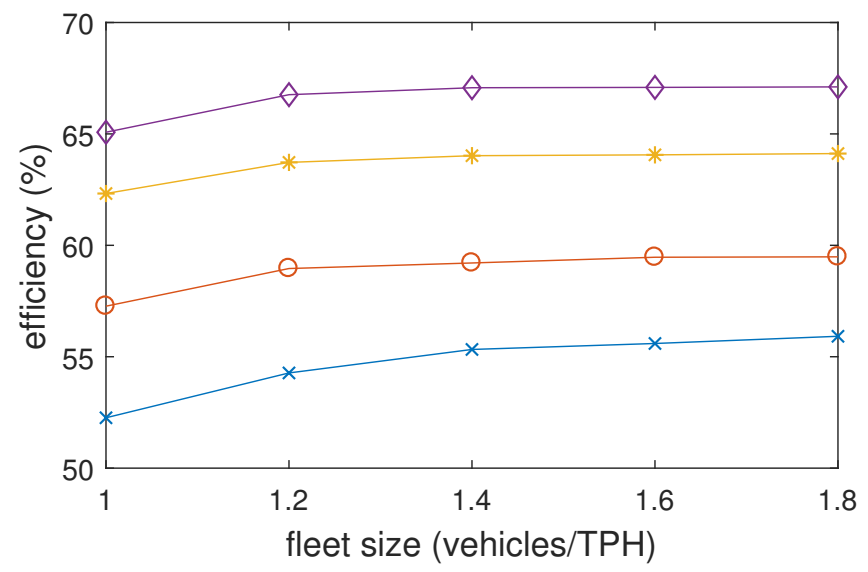

(b)

Figure 7: System performance parameters as a function of the number of SAEVs and trips per hour (tph). (a) Break-even prices; (b) efficiency

quests). Overall, BEP decreases as the TPH increase, together with the increase in efficiency of the system (Fig. 7b). For 1000 TPH and a fleet size of 1200 vehicles the BEP is about $30 \mathrm{JPY} / \mathrm{km}(0.27 \mathrm{USD} / \mathrm{km})$, about 10 times lower than the average Japanese standard taxi fare of about $300 \mathrm{JPY} / \mathrm{km}$ 
(2.70 USD $/ \mathrm{km})$ [56]. This is a price comparable with public transport fares.

The efficiency of the system tends to increase with larger fleets, thanks to a more ubiquitous presence of vehicles to satisfy transport demand without extra empty trips (Fig. 7c). However, as with waiting times, efficiency also tends to reach a plateau, the value of which is a function of the total TPH. Higher TPH values are associated with higher overall efficiency of the system. Rejected requests drop to zero for fleet sizes larger than a threshold size, which is dependent on the total TPH (Table 5).

Table 5: Requests served

\begin{tabular}{l|lllll} 
& \multicolumn{5}{|c}{ vehicles/TPH } \\
$\mathrm{TPH}$ & 1 & 1.2 & 1.4 & 1.6 & 1.8 \\
\hline 100 & $95.65 \%$ & $99.64 \%$ & $99.98 \%$ & $99.99 \%$ & $100 \%$ \\
200 & $98.16 \%$ & $99.95 \%$ & $99.99 \%$ & $99.99 \%$ & $100 \%$ \\
500 & $99.70 \%$ & $99.99 \%$ & $100 \%$ & $100 \%$ & $100 \%$ \\
1000 & $99.79 \%$ & $99.99 \%$ & $100 \%$ & $100 \%$ & $100 \%$
\end{tabular}

Results show that the system is able to operate efficiently with between 1.2 and 1.6 vehicles per trip per hour, or about 5 to 7 vehicles per 100 trips per day. Assuming an average of 2 trips per private vehicle per day, this suggests that autonomous vehicles can replace private vehicles with a proportion of about 1:7 to 1:10, depending on the expected quality of service (waiting times and prices), in accordance with previous studies as discussed in section 2 . The results also demonstrate the feasibility of the system even without planned active re-balancing of the vehicles. However, with an effective re-balancing strategy waiting time can be reduced further. 


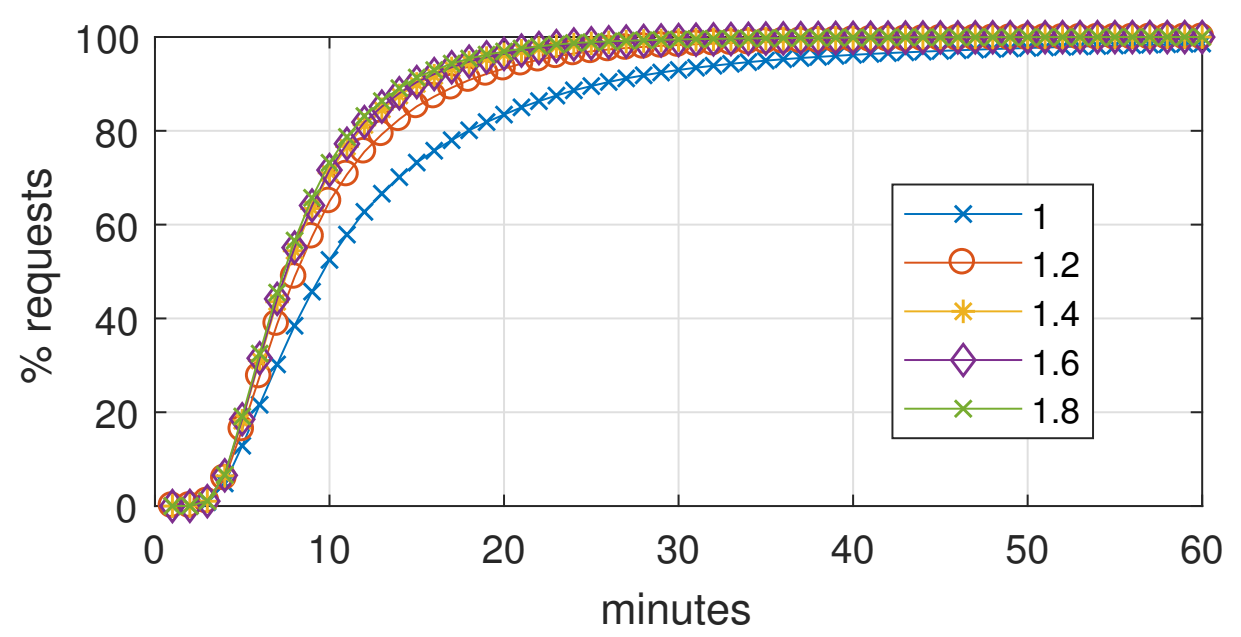

Figure 8: Cumulative waiting time distribution for simulations with 1000 TPH and 1 to 1.8 vehicles per TPH.

\subsection{Charge scheduling algorithm}

The influence of electricity pricing was investigated by testing the model with the price profiles introduced in section 3.10. Fig. 9 shows the results of different charging strategies with the different price profiles. In all the simulations there are no rejected requests and the waiting times are the same as those found in section 4.1, thus demonstrating that the charge scheduling algorithm has no negative effect on the transport service quality.

The charge scheduling algorithm lowers the BEP, with the benefits substantially higher when employing V2G. The non-V2G strategy differs only in that the vehicle can not sell back to the grid, thus only the positive values of (8) are considered. The savings are particularly significant with highly volatile price profiles such as profile 3 . V2G can therefore play a role in making the system more economically viable. In a scenario with 


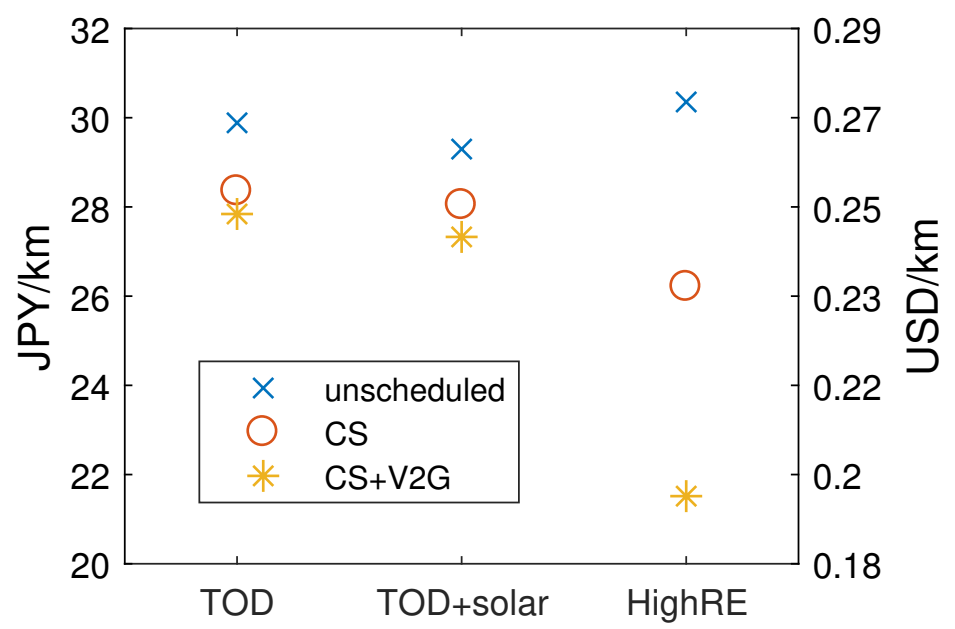

Figure 9: BEP for different charging strategies and price profiles. Simulation with 1000 trips per hour over 20 days.

rapidly changing electricity prices, that is, with a high penetration of nondispatchable generation, under the assumptions of this work V2G has the potential to significantly decrease the energy costs for the system and help to balance the grid.

\subsection{Operating reserve capacity}

The performance of the system as a supplier of operating reserve is tested with the model introduced in section 3.5. Two types of operating reserve were tested: spinning reserve, modeled with requests with no allowed delay; and non-spinning reserve, modeled with an allowed delay of 15 minutes. In both cases, the capacity of the system was tested for different request duration of 30 and 60 minutes. Two types of grid connection for peak power were also tested: $20 \mathrm{~kW}$ and $50 \mathrm{~kW}$. Moreover, the possibility for the system to be used to supply reserve storage was also tested in the same way. The secondary 
objective weight in (23) was chosen as 0.001. This is an arbitrarily small value in order not to affect the main optimization, while still allowing for the optimization of the use of vehicles for the same amount of power delivered. The results would not change as long as the secondary objective weight is small enough.

The available power varies significantly depending on the time of day, due to variable number of vehicles connected to charging stations and the available SOC. The limiting period was identified as the peak transport demand between 8 and 9 in the morning. The system was therefore tested for this period, to calculate the minimum power available. The tests were run at 10 minutes intervals for 50 times.

Fig. 10 and 11 show the results with a maximum power connection of 20 $\mathrm{kW}$ and duration of 30 minutes. The results of the tests with a duration of 60 minutes were the same as for the 30 minutes, thus are not shown. The allowed delay is the most significant factor in determining the amount of available power for a $20 \mathrm{~kW}$ connection: when 15 minutes delays are allowed, the power available grows by about 1-1.5 MW per 1000 vehicles. This results from the fact that the limiting factor in this case is the power deliverable (that is, the number of vehicles connected to charging stations), and not the energy stored in the batteries. A longer allowed delay allows more vehicles to move to charging stations to contribute during an operating reserve request. This conclusion is supported by the results of the $50 \mathrm{~kW}$ connection in Fig. 12 , which are almost exactly increased by a factor $50 / 20$.

Figures 13-14 show the results for negative operating reserve capacity, or to absorb excess generation (storage). This service may become relevant as 


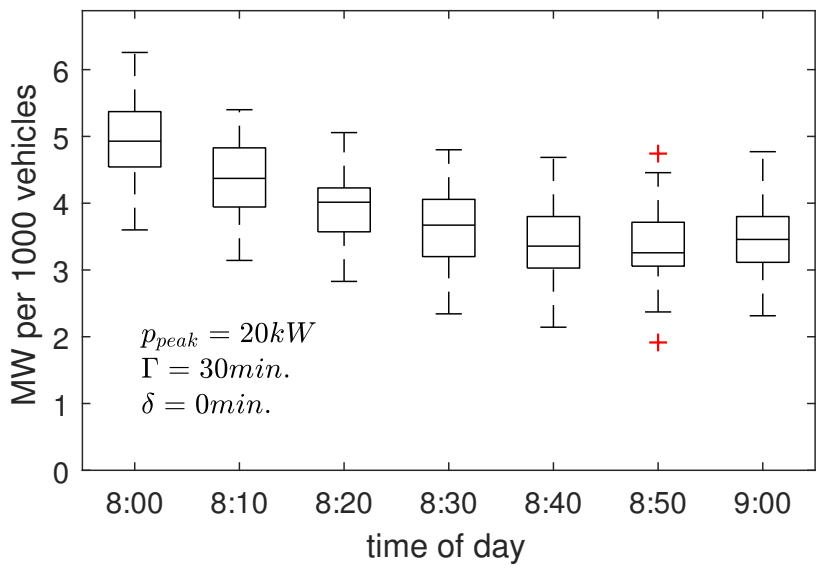

Figure 10: Results of 50 tests for operating reserve capacity with no delay for 30 minutes. Results for 60 minutes duration were found to be the same. Whiskers in the boxplot include the upper and lower values up to 1.5 IQR distance from the third and first quartiles, respectively. Red plus signs indicate outliers.

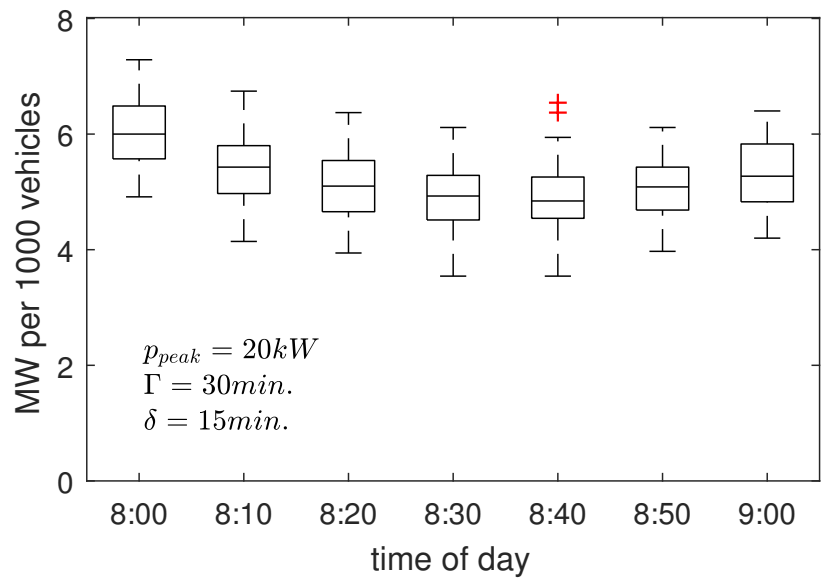

Figure 11: Results of 50 tests for operating reserve capacity with 15 minutes delay for 30 minutes. Results for 60 minutes duration were found to be the same.

penetration of intermittent renewable energy increase.

The impact on the transport service was also investigated. In Fig. 15 the 


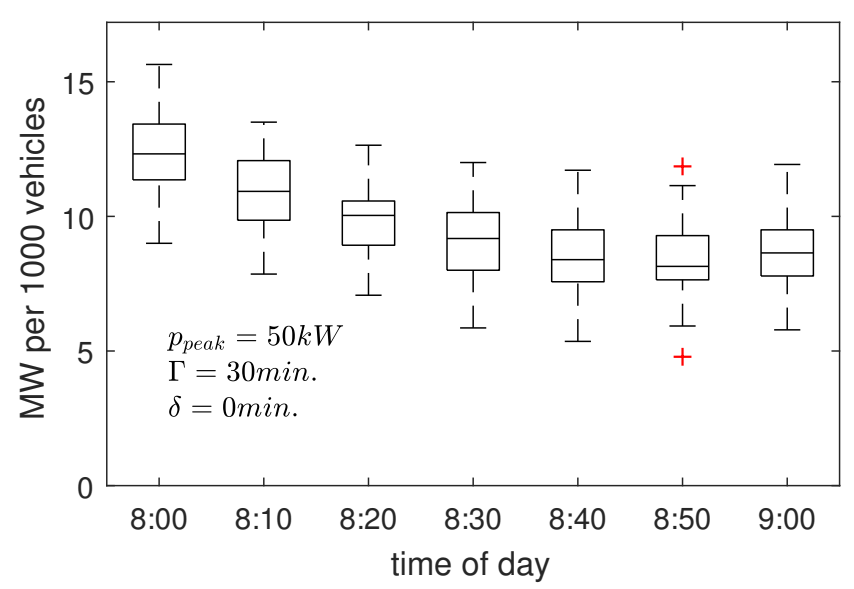

Figure 12: Results of 50 tests for operating reserve capacity with no delay for 30 minutes, with a $50 \mathrm{~kW}$ connection

average waiting times are shown for a 30 minutes non-spinning request in the morning peak and at the afternoon peak. Note that the 15 minutes allowed delay is the worst case scenario since with no allowed delay the vehicles participating in the request will necessarily be fewer.

Providing operating reserve with $20 \mathrm{~kW}$ connection does not influence the request rejection rate, and momentarily increase the average waiting time for passengers during peak times.

\section{Conclusions}

A simulation model was developed in MATLAB ${ }^{\mathrm{TM}}$ to study the feasibility of a shared autonomous electric vehicle transport system and its integration with the electricity grid, in particular its potential to supply operating reserve. The model simulates a SAEV fleet serving passengers and charging at designated charging stations. A charge scheduling algorithm based on 


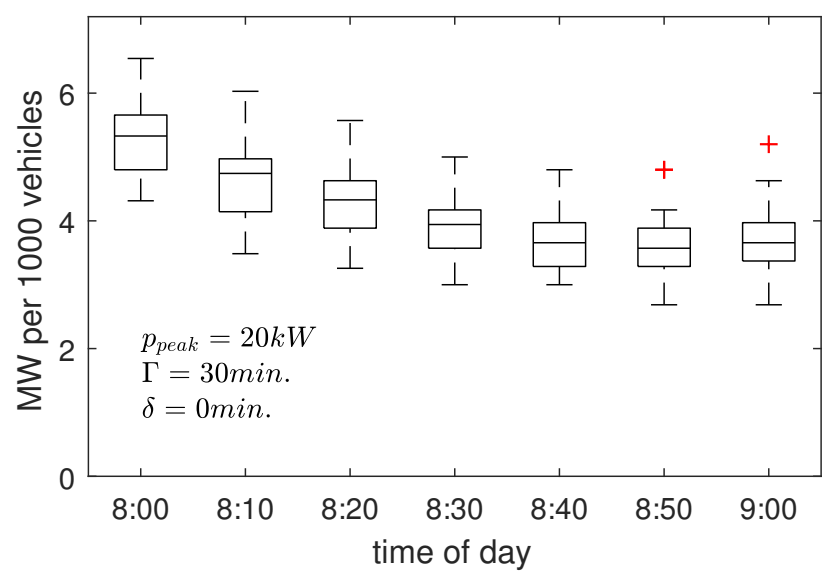

Figure 13: Results of 50 tests for negative operating reserve capacity (storage of excess generation) with no delay for 30 minutes, with a $20 \mathrm{~kW}$ connection

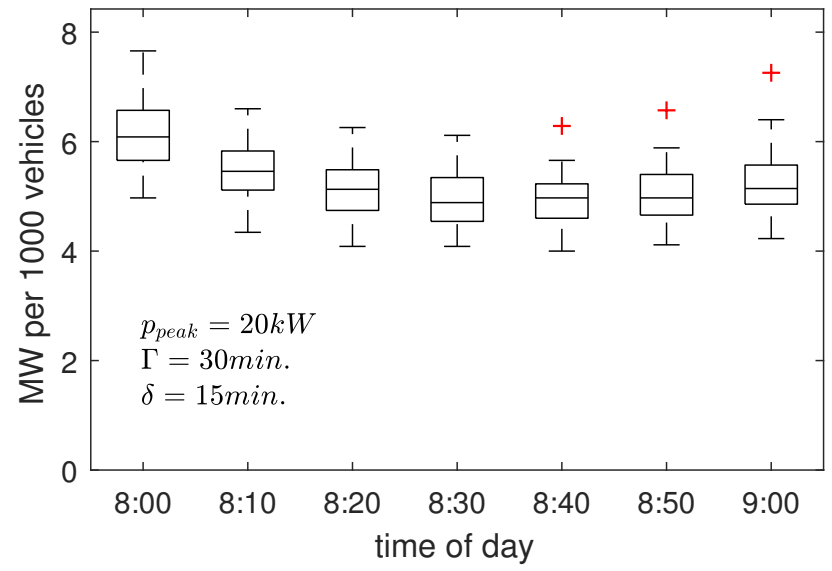

Figure 14: Results of 50 tests for negative operating reserve capacity (storage of excess generation) with 15 minutes delay for 30 minutes, with a $20 \mathrm{~kW}$ connection

electricity prices was used and tested with several price profiles.

The city of Tokyo was taken as a case study, with passenger data based on a transport survey. The system is studied in a transitional phase, with the assumption of unchanged transport demand patterns and a limited number 


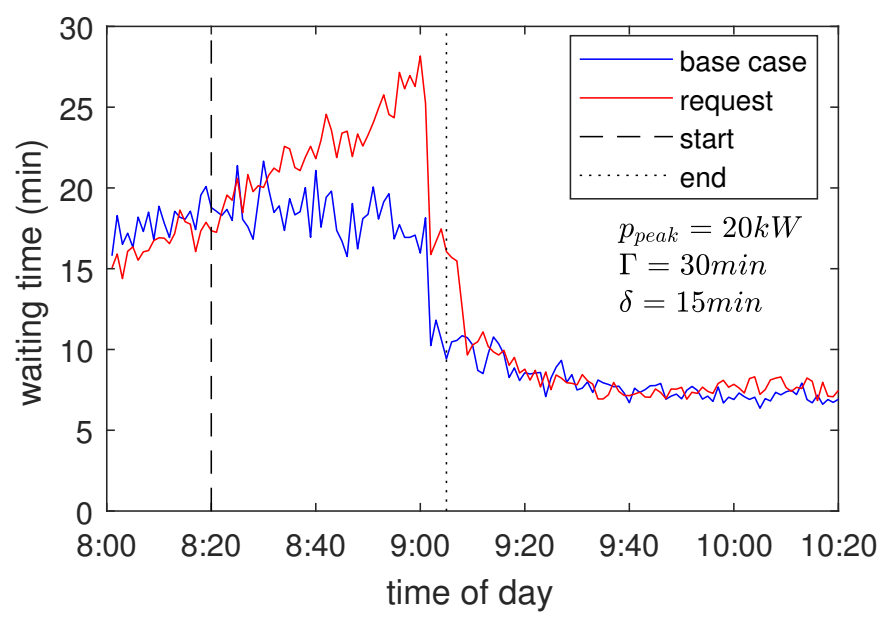

(a)

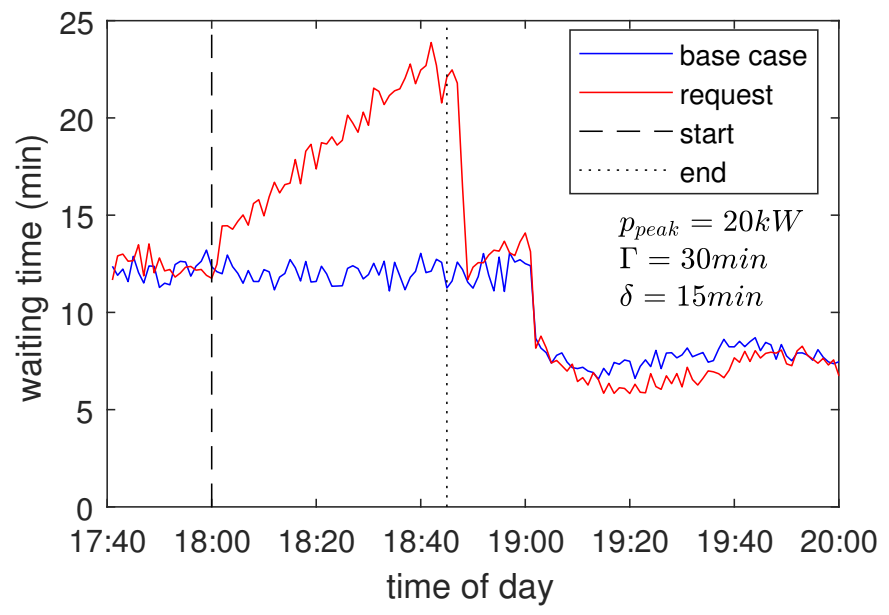

(b)

Figure 15: Average waiting times for base case and for a case with a 30 minutes non-spinning operating reserve request. (a) morning peak; (b) afternoon peak. Average over 10 simulations for each case. For all the simulations, there were no rejected requests. 
of vehicles, and assuming that the power flow of vehicles does not influence electricity prices or transmission congestion levels.

The results of the simulations show that every shared vehicle in a fleet of SAEVs in Tokyo could replace 7 to 10 private cars, depending on the trade-off between waiting time and cost of the system. The system's break-even price per $\mathrm{km}$ is significantly lower than the fare of traditional taxis, and comparable to the average cost of car ownership and public transport. The results also suggests that the integration of a charge scheduling algorithm can further lower the cost of transport by providing load shifting and storage for the grid. In the case of highly volatile price profiles, the break-even prices of the system drop by up to $40 \%$ thanks to charge scheduling with vehicle-to-grid.

The model also suggested the viability of SAEVs for providing spinning and non-spinning operating reserve to the grid. The amount of operating reserve power available depends strongly on the time of day and the allowed delay. In particular, the system is able to supply spinning reserve of up to about 3.5 MW per 1000 vehicles even at the worst time for 1 hour with a 20 kW connection. This increase to 8-9 MW per 1000 vehicles when using a 50 $\mathrm{kW}$ connection.

In a scenario of a wide implementation of this system, our model suggest that SAEVs could provide significant grid-scale storage and spinning reserves. However, to assess the real impact of this technology implemented at large scale, a dynamic transport model needs to be developed to account for the change in transport patterns and congestion levels. Moreover, a model of the electricity transmission network could be included in future work to account for transmission constraints for a full-scale system. 


\section{Acknowledgments}

The authors would like to thank the Ministry of Land, Infrastructure, Transport and Tourism of Japan for the access to the transport survey data.

\section{References}

[1] B. Boyacı, K. G. Zografos, N. Geroliminis, An optimization framework for the development of efficient one-way car-sharing systems, European Journal of Operational Research 240 (3) (2015) 718-733. doi:10.1016/ j.ejor. 2014.07 .020 .

[2] J. B. Greenblatt, S. Shaheen, Automated Vehicles, On-Demand Mobility, and Environmental Impacts, Current Sustainable/Renewable Energy Reports 2 (3) (2015) 74-81. doi:10.1007/s40518-015-0038-5.

[3] D. J. Fagnant, K. M. Kockelman, The travel and environmental implications of shared autonomous vehicles, using agent-based model scenarios, Transportation Research Part C: Emerging Technologies 40 (2014) 1-13. doi:10.1016/j.trc.2013.12.001.

[4] S. Rand Transportation, and Technology (Program), Autonomous Vehicle Technology: a Guide for Policymakers, 2014.

[5] S. Hörl, F. Ciari, K. W. Axhausen, Recent perspectives on the impact of autonomous vehicles.

URL https://www.ethz.ch/content/dam/ethz/special-interest/ baug/ivt/ivt-dam/vpl/reports/2016/ab1216.pdf 
[6] J. B. Greenblatt, S. Saxena, Autonomous taxis could greatly reduce greenhouse-gas emissions of US light-duty vehicles, Nature Climate Change 5 (9) (2015) 860-863. doi:10.1038/nclimate2685.

[7] T. D. Chen, K. M. Kockelman, J. P. Hanna, Operations of a shared, autonomous, electric vehicle fleet: Implications of vehicle \& charging infrastructure decisions, Transportation Research Part A: Policy and Practice 94 (2016) 243-254. doi:10.1016/j.tra.2016.08.020.

URL http://linkinghub.elsevier.com/retrieve/pii/ S096585641630756X

[8] Timothy Sweda, Diego Klabjan, An Agent-Based Decision Support System for Electric Vehicle Charging Infrastructure Deployment, IEEE, Piscataway, NJ, 2011.

[9] Z. Wadud, D. MacKenzie, P. Leiby, Help or hindrance? The travel, energy and carbon impacts of highly automated vehicles, Transportation Research Part A: Policy and Practice 86 (2016) 1-18. doi:10.1016/j. tra.2015.12.001.

[10] J. Weiss, R. Hledik, R. Lueken, T. Lee, W. Gorman, The electrification accelerator: Understanding the implications of autonomous vehicles for electric utilities, The Electricity Journal 30 (10) (2017) 50-57. doi:10.1016/j.tej.2017.11.009.

URL http://linkinghub.elsevier.com/retrieve/pii/ S1040619017303093

[11] D. B. Richardson, Electric vehicles and the electric grid: A review 
of modeling approaches, Impacts, and renewable energy integration, Renewable and Sustainable Energy Reviews 19 (2013) 247-254. doi: $10.1016 / j . r s e r .2012 .11 .042$.

[12] D. Dallinger, M. Wietschel, Grid integration of intermittent renewable energy sources using price-responsive plug-in electric vehicles, Renewable and Sustainable Energy Reviews 16 (5) (2012) 3370-3382.

URL https://econpapers.repec.org/article/eeerensus/v_3a16_ 3ay_3a2012_3ai_3a5_3ap_3a3370-3382.htm

[13] W. Kempton, J. Tomić, Vehicle-to-grid power implementation: From stabilizing the grid to supporting large-scale renewable energy, Journal of Power Sources 144 (1) (2005) 280-294. doi:10.1016/j.jpowsour. 2004.12 .022 .

[14] M. Yilmaz, P. T. Krein, Review of the Impact of Vehicle-to-Grid Technologies on Distribution Systems and Utility Interfaces, IEEE Transactions on Power Electronics 28 (12) (2013) 5673-5689. doi: 10.1109/TPEL . 2012.2227500.

[15] K. Clement-Nyns, E. Haesen, J. Driesen, The Impact of Charging PlugIn Hybrid Electric Vehicles on a Residential Distribution Grid, IEEE Transactions on Power Systems 25 (1) (2010) 371-380. doi:10.1109/ TPWRS . 2009. 2036481.

[16] C. Fernandes, P. Frías, J. M. Latorre, Impact of vehicle-to-grid on power system operation costs: The Spanish case study, Applied Energy 96 (2012) 194-202. doi:10.1016/j.apenergy.2011.11.058. 
[17] B. Tarroja, B. Shaffer, S. Samuelsen, The importance of grid integration for achievable greenhouse gas emissions reductions from alternative vehicle technologies, Energy 87 (2015) 504-519. doi:10.1016/j.energy.2015.05.012.

URL http://www.sciencedirect.com/science/article/pii/ S0360544215005460

[18] J. D. K. Bishop, C. J. Axon, D. Bonilla, D. Banister, Estimating the grid payments necessary to compensate additional costs to prospective electric vehicle owners who provide vehicle-to-grid ancillary services, Energy 94 (2016) 715-727. doi:10.1016/j.energy.2015.11.029.

[19] Burns, Jordan, Scarborough, Transforming Personal Mobility, Tech. rep. (2013).

[20] S. Hörl, Implementation of an autonomous taxi service in a multi-modal traffic simulation using MATSim (2016).

URL http://studentarbeten.chalmers.se

[21] J. Liu, K. M. Kockelman, P. M. Boesch, F. Ciari, Tracking a system of shared autonomous vehicles across the Austin, Texas network using agent-based simulation, Transportation 44 (6) (2017) 1261-1278. doi: 10.1007/s11116-017-9811-1.

URL http://link.springer.com/10.1007/s11116-017-9811-1

[22] W. Zhang, S. Guhathakurta, J. Fang, G. Zhang, Exploring the impact of shared autonomous vehicles on urban parking demand: An agent-based 
simulation approach, Sustainable Cities and Society 19 (2015) 34-45. doi:10.1016/j.scs.2015.07.006.

[23] K. Spieser, K. Treleaven, R. Zhang, E. Frazzoli, D. Morton, M. Pavone, Toward a Systematic Approach to the Design and Evaluation of Automated Mobility-on-Demand Systems: A Case Study in Singapore, in: G. Meyer, S. Beiker (Eds.), Road Vehicle Automation, Springer International Publishing, Cham, 2014, pp. 229-245.

[24] M. W. Levin, K. M. Kockelman, S. D. Boyles, T. Li, A general framework for modeling shared autonomous vehicles with dynamic network-loading and dynamic ride-sharing application, Computers, Environment and Urban Systems 64 (2017) 373-383. doi:10.1016/j. compenvurbsys. 2017.04.006.

URL http://linkinghub.elsevier.com/retrieve/pii/ S019897151630237X

[25] M. Pavone, S. L. Smith, E. Frazzoli, D. Rus, Robotic load balancing for mobility-on-demand systems, The International Journal of Robotics Research 31 (7) (2012) 839-854. doi:10.1177/0278364912444766.

[26] M. Volkov, J. Aslam, D. Rus, Markov-based redistribution policy model for future urban mobility networks, in: Intelligent Transportation Systems (ITSC), 2012 15th International IEEE Conference on, IEEE, 2012, pp. 1906-1911.

URL http://ieeexplore.ieee.org/abstract/document/6338848/

[27] F. Acquaviva, D. D. Paola, A. Rizzo, A novel formulation for the 
distributed solution of load balancing problems in mobility on-demand systems, in: 2014 International Conference on Connected Vehicles and Expo (ICCVE), 2014, pp. 906-911. doi:10.1109/ICCVE. 2014. 7297687.

[28] R. Zhang, F. Rossi, M. Pavone, Model predictive control of autonomous mobility-on-demand systems, in: 2016 IEEE International Conference on Robotics and Automation (ICRA), 2016, pp. 1382-1389. doi:10. 1109/ICRA. 2016.7487272.

[29] E. S. Rigas, S. D. Ramchurn, N. Bassiliades, Algorithms for Electric Vehicle Scheduling in Mobility-on-Demand Schemes, in: 2015 IEEE 18th International Conference on Intelligent Transportation Systems, 2015, pp. 1339-1344. doi:10.1109/ITSC.2015.220.

[30] E. Biondi, C. Boldrini, R. Bruno, The impact of regulated electric fleets on the power grid: The car sharing case, in: 2016 IEEE 2nd International Forum on Research and Technologies for Society and Industry Leveraging a better tomorrow (RTSI), 2016, pp. 1-6. doi: 10.1109/RTSI . 2016.7740570.

[31] D. Freund, A. F. Raab, T. Küster, S. Albayrak, K. Strunz, Agentbased integration of an electric car sharing fleet into a smart distribution feeder, in: Innovative Smart Grid Technologies (ISGT Europe), 2012 3rd IEEE PES International Conference and Exhibition on, IEEE, 2012, pp. $1-8$.

[32] J. Bischoff, M. Maciejewski, Agent-based Simulation of Electric Taxicab 
Fleets, Transportation Research Procedia 4 (2014) 191-198. doi:10. 1016/j.trpro.2014.11.015.

[33] H. Wang, R. Cheu, Operations of a taxi fleet for advance reservations using electric vehicles and charging stations, Transportation Research Record: Journal of the Transportation Research Board (2352) (2013) $1-10$.

URL http://trrjournalonline.trb.org/doi/abs/10.3141/ 2352-01

[34] F. Mwasilu, J. J. Justo, E.-K. Kim, T. D. Do, J.-W. Jung, Electric vehicles and smart grid interaction: A review on vehicle to grid and renewable energy sources integration, Renewable and Sustainable Energy Reviews 34 (2014) 501-516. doi:10.1016/j.rser.2014.03. 031.

[35] P. Nunes, M. C. Brito, Displacing natural gas with electric vehicles for grid stabilization, Energy 141 (2017) 87-96. doi:10.1016/j.energy.2017.09.064.

URL http://www.sciencedirect.com/science/article/pii/ S0360544217315852

[36] H. W. Kuhn, The Hungarian method for the assignment problem, Naval Research Logistics Quarterly 2 (1-2) (1955) 83-97. doi:10.1002/nav. 3800020109 .

[37] W. J. Mitchell, C. Borroni-Bird, L. D. Burns, Reinventing the au- 
tomobile personal urban mobility for the 21st century, Massachusetts Institute of Technology, Cambridge, Mass., 2010.

[38] Q. Wang, C. Zhang, Y. Ding, G. Xydis, J. Wang, J. Ostergaard, Review of real-time electricity markets for integrating Distributed Energy Resources and Demand Response, Applied Energy 138 (2015) 695-706. doi:10.1016/j . apenergy . 2014.10.048.

[39] Japan Electric Power Information Center, Inc. (JEPIC), Operating and Financial Data (2015).

URL https://www.jepic.or.jp/en/data/japan_data.pdf

[40] Ministry of Land, Infrastructure, Transport and Tourism, Japan (MLIT), Tokyo Metropolitan Area Person trip survey (2008).

[41] Tokyo Statistical Yearbook 2009-2015 (2016).

URL http: //www.toukei.metro.tokyo.jp/tnenkan/tn-eindex.htm

[42] Developer's Guide | Google Maps Geocoding API.

URL https://developers.google.com/maps/documentation/ geocoding/intro

[43] Tokyo Metropolitan Government Environment Bureau, Traffic volume and traffic speed in Tokyo.

URL http://www.kankyo.metro.tokyo.jp/en/attachement/c-3_ data.pdf

[44] Transitions to Alternative Vehicles and Fuels, National Academies Press, Washington, D.C., 2013. doi:10.17226/18264.

URL http://www . nap.edu/catalog/18264 
[45] 2013 Nissan Leaf Advanced Vehicle Testing - Baseline Testing Results (2013).

URL https://energy.gov/sites/prod/files/2015/01/f19/ fact2013nissanleaf .pdf

[46] X. Han, M. Ouyang, L. Lu, J. Li, A comparative study of commercial lithium ion battery cycle life in electric vehicle: Capacity loss estimation, Journal of Power Sources 268 (2014) 658-669. doi:10.1016/j. jpowsour.2014.06.111.

[47] MaxRange Tesla Battery Survey.

URL goo.gl/JCLJOE

[48] Tesla Inc., Tesla Powerwall Limited Warranty (USA) (Jun. 2016).

[49] K. Uddin, T. Jackson, W. D. Widanage, G. Chouchelamane, P. A. Jennings, J. Marco, On the possibility of extending the lifetime of lithium-ion batteries through optimal V2g facilitated by an integrated vehicle and smart-grid system, Energy 133 (2017) 710-722. doi: 10.1016/j.energy.2017.04.116.

[50] IHS Automotive, Emerging technologies: autonomous cars- not if, but when (2014).

[51] D. J. Fagnant, K. Kockelman, Preparing a nation for autonomous vehicles: opportunities, barriers and policy recommendations, Transportation Research Part A: Policy and Practice 77 (2015) 167-181. doi:10.1016/j.tra.2015.04.003. 
[52] B. Nykvist, M. Nilsson, Rapidly falling costs of battery packs for electric vehicles, Nature Climate Change 5 (4) (2015) 329-332. doi:10.1038/ nclimate2564.

[53] I. J. Fernández, C. F. Calvillo, A. Sánchez-Miralles, J. Boal, Capacity fade and aging models for electric batteries and optimal charging strategy for electric vehicles, Energy 60 (2013) 35-43. doi:10.1016/j . energy . 2013.07.068.

URL http://www.sciencedirect.com/science/article/pii/ S0360544213006749

[54] S. Han, S. Han, H. Aki, A practical battery wear model for electric vehicle charging applications, Applied Energy 113 (2014) 1100-1108. doi:10.1016/j.apenergy.2013.08.062.

URL http://linkinghub.elsevier.com/retrieve/pii/ S030626191300706X

[55] TEPCO : Customer Communication | Rate Calculation.

URL http://www.tepco.co.jp/en/customer/guide/ratecalc-e. html

[56] Order a Cab (Rates Table) ? Tokyo Taxi [Tokyo Hire-Taxi Association(THTA)].

URL http://www.taxi-tokyo.or.jp/english/call/pricelist. html 